\title{
Intra-operative 3D guidance and edema detection in prostate brachytherapy using a non-isocentric C-arm
}

\author{
A. Jain ${ }^{a, c},{ }^{\star}$, A. Deguet ${ }^{a}$, I. Iordachita $^{a}$, G. Chintalapani $^{a}$, S. Vikal ${ }^{d}$, J. Blevins $^{b}$, Y. Le $^{a}$, E. \\ Armour $^{\mathrm{a}}$, C. Burdette ${ }^{\mathrm{b}}$, D. Song ${ }^{\mathrm{a}}$, and G. Fichtinger ${ }^{\mathrm{a}, \mathrm{d}}$ \\ aJohns Hopkins University, 3400 N Charles St, Baltimore, MD 21210, United States \\ bAcoustic MedSystems Inc., 206 Randolph St Suite 301, Champaign, IL 61820, United States \\ cPhilips Research North America, 345 Scarborough Road, NY 10510, United States \\ 'Queen's University, 725 Goodwin Hall, Kingston, ON, Canada K7L 3N6
}

\section{Abstract}

Purpose-Brachytherapy (radioactive seed insertion) has emerged as one of the most effective treatment options for patients with prostate cancer, with the added benefit of a convenient outpatient procedure. The main limitation in contemporary brachytherapy is faulty seed placement, predominantly due to the presence of intra-operative edema (tissue expansion). Though currently not available, the capability to intra-operatively monitor the seed distribution, can make a significant improvement in cancer control. We present such a system here.

Methods-Intra-operative measurement of edema in prostate brachytherapy requires localization of inserted radioactive seeds relative to the prostate. Seeds were reconstructed using a typical nonisocentric C-arm, and exported to a commercial brachytherapy treatment planning system. Technical obstacles for 3D reconstruction on a non-isocentric C-arm include pose-dependent Carm calibration; distortion correction; pose estimation of $\mathrm{C}$-arm images; seed reconstruction; and C-arm to TRUS registration.

Results-In precision-machined hard phantoms with 40-100 seeds and soft tissue phantoms with 45-87 seeds, we correctly reconstructed the seed implant shape with an average 3D precision of $0.35 \mathrm{~mm}$ and $0.24 \mathrm{~mm}$, respectively. In a DoD Phase- 1 clinical trial on six patients with 48-82 planned seeds, we achieved intra-operative monitoring of seed distribution and dosimetry, correcting for dose inhomogeneities by inserting an average of over four additional seeds in the six enrolled patients (minimum 1; maximum 9). Additionally, in each patient, the system automatically detected intra-operative seed migration induced due to edema (mean $3.84 \mathrm{~mm}$, STD $2.13 \mathrm{~mm}$, Max $16.19 \mathrm{~mm}$ ).

Conclusions-The proposed system is the first of a kind that makes intra-operative detection of edema (and subsequent re-optimization) possible on any typical non-isocentric C-arm, at negligible additional cost to the existing clinical installation. It achieves a significantly more

"Corresponding author at: Johns Hopkins University, 3400 N Charles St, Baltimore, MD 21210, United States. Ameet.Jain@Philips.com (A. Jain). 
homogeneous seed distribution, and has the potential to affect a paradigm shift in clinical practice.

Large scale studies and commercialization are currently underway.

\section{Keywords}

Fluoroscopic guidance; Prostate brachytherapy; Low cost; Edema; 3D Reconstruction

\section{Introduction}

With an annual incidence of over 200,000 new cases and 27,000 deaths in the US, prostate cancer continues to be the most common cancer in men (Jemal et al., 2004), and is expected to double its incidence rates by 2015 . Currently one in every six men get diagnosed with it at some point in their life. The American Cancer Society estimates that in 2015 there would be 450,000 new cases of prostate cancer diagnosed each year, roughly doubling the current incidence. For several decades, the definitive treatment for low risk prostate cancer was radical prostatectomy (complete prostate removal) or external beam radiation therapy (Peschel and Colberg, 2003). It has been known for several years now that low dose rate permanent seed brachytherapy (shortly brachytherapy thereafter in this document) today can achieve virtually equivalent outcomes (Blasko et al., 2002). Brachytherapy is a one-time outpatient procedure, without the surgical trauma of prostatectomy or the grinding routine of external beam radiation therapy. In this treatment a large number of small cylindrical ( $1 \mathrm{~mm}$ diameter; $5 \mathrm{~mm}$ length) radioactive capsules are implanted into the prostate to kill the cancer by emitting radiation. It was first performed in 1911 with limited success. Brachytherapy is typically performed transperineally under real-time transrectal ultrasound (TRUS) guidance. $\mathrm{C}$-arm fluoroscopy or X-ray images are often used for gross visual observation of the implant. If accurately executed, it can achieve a sharp demarcation between the treated volume and healthy structures, and thereby achieve superior tumor control with reduced morbidity (Talcott et al., 2003).

According to CaPSURE, a national disease registry, a steadily growing proportion of prostate cancer patients with low risk disease have seen a major shift in cancer management (Cooperberg et al., 2004). Recent estimates indicate a large increase in the number of brachytherapy procedures, with $30-40 \%$ of all prostate cancer patients receiving brachytherapy as part of their treatment (Grimm and Sylvester, 2004), with an overall 15year survival rate of $74 \%$ (88\%, 80\% and 53\% in low, intermediate and high-risk patients). By conservative linear extrapolation of the CaPSURE data, at least 40,000 brachytherapy procedures were expected to be performed in the US in 2005.

\subsection{Limitations in contemporary practice}

Intra-operative tissue expansion (edema) and faulty needle and seed placement often cause insufficient dose to the cancer and/or inadvertent radiation of the rectum, urethra, and bladder. Seed implant planning is based on idealistic pre-planned patterns that, as fifteen years of clinical practice has clearly demonstrated, are not achievable in the actual human body. According to a comprehensive review by the American Brachytherapy Society (Nag et al., 2001), the pre-planned technique used for permanent prostate brachytherapy has limitations that may be overcome by intra-operative planning. At the same time, continues 
the report, the major current limitation of intra-operative planning is the inability to localize the seeds in relation to the prostate. Though excellent treatment computation tools are available today (Polo et al., 2010), these methods, critically require that the exact 3D locations of the implanted seeds be precisely known with respect to the patient's anatomy. Unfortunately, individual seeds cannot be currently localized intra-operatively, since seeds are not well visible in ultrasound images (Han et al., 2003). Furthermore, edema is a natural response of the body, wherein the tissue expands due to the needle-insertions and radiation, temporarily increasing the prostate volume by up to $100 \%$ (mean half-life of 9.3 days) and decreasing the dose to the tissue. Also many patients suffer from side-effects like secondary malignancies, urinary symptoms, rectal bleeding, and sexual impotency (Grimm and Sylvester, 2004).

\subsection{Alternative technologies}

The success of brachytherapy (i.e. maximizing its curative force while minimizing its comorbidity) chiefly depends on our ability to tailor the therapeutic dose to the patient's individual anatomy. Virtually all studies agree that the next paradigm shift is expected to be the capability to intra-operatively monitor the inserted seeds. The biggest bottleneck toward this, is seed migration, making intra-operative monitoring of seed distribution the holy grail.

1.2.1. TRUS imaging - TRUS imaging is almost exclusively the imaging modality of choice in Prostate brachytherapy. TRUS has been overwhelmingly popular for needle guidance thanks to its real-time nature, lowcost, and apparent ease of use. However, it has limited success when investigated for quantitative intra-operative dosimetry. While TRUS provides adequate imaging of the soft tissue anatomy, it does not allow for robust localization of the implanted seeds. Various researchers have tried to segment the seeds from TRUS images by a variety of methods (Mitri et al., 2009b; Xue et al., 2004; Dumane et al., 2004; Tornes and Eriksen, 2003; Holmes et al., 2003; French et al., 2004), but even when meticulously hand-segmented, $25 \%$ of the seeds may remain hidden in ultrasound (Han et al., 2003). It seems that this technique alone cannot become clinically feasible for intra-operative seed localization.

1.2.2. Image-guided robots-Image-guided robots have been developed (Fichtinger et al., 2006; Wei et al., 2004) for brachytherapy. These systems are accurate only in image coordinates, but are prone to miss the target anatomy due to tissue deformation and needle bending. Modeling tissue deformation is potentially useful, but clinically practical results are not expected to arrive any time soon. Moreover, accurate seed placement using robots does not solve the issue of seed migration, and hence would still need the ability to intraoperatively compute the location of the seeds.

1.2.3. Invasive surgical fixation-Invasive surgical fixation of the prostate with 2-3 needles locking the prostate (Taschereau et al., 2000) has been undoubtedly useful, but it alone cannot guarantee optimal implants. The extra needles increase tissue damage and the likelihood of edema, but do not increase the seed placement accuracy. It is therefore imperative that both seed placement and migration errors be compensated during the implant procedure. 
1.2.4. Intra-operative $\mathbf{C T}$, MRI-Intra-operative CT, MRI have been used by a handful of practitioners, either for direct guidance (Koutrouvelis et al., 2003; Molloy et al., 1999), or for dosimetry (Kaplan et al., 2006). For routine use, CT is impractical because (1) it is not a true real-time tool, (2) is not available in the OR, and (3) exposes the patient and operating team to ionizing radiation. MRI-guided brachytherapy (D'Amico et al., 2000; Hurwitz et al., 2000 ) is prohibitively expensive for routine clinical use and only a handful of patients could benefit from this technology in the foreseeable future.

1.2.5. C-arm fluoroscopy-C-arm fluoroscopy, as has been suggested by the published history, originates (Kumar et al., 1985) when it was first used as a solo guidance modality. Shortly after TRUS emerged as a primary image guidance modality, fluoroscopy has become a secondary tool for gross visual observation. Mobile C-arms are ubiquitous in contemporary prostate brachytherapy, with approximately $60 \%$ of the brachytherapy practitioners use it (Prestidge et al., 1998). It is considered the gold standard for intraoperative visualization of brachytherapy seeds. Unfortunately, it can currently be used only for qualitative implant analysis and not for providing any quantitative intra-operative dosimetry guidance. Cone-beam computed tomography has been explored (Siewerdsen et al., 2007), though it imparts high radiation doses and is more expensive. Moreover, in a typical setup the C-arm's motion is constrained to less than $15^{\circ}$ around the AP-axis. A continuous tomosynthesis reconstruction (Brunet-Benkhoucha et al., 2009; Tutar et al., 2003) could be of potential use, though the published literature does not yet report any method that is sufficiently robust for routine clinical use.

\subsection{The proposed solution - C-arm to TRUS fusion}

As can be seen, currently there is no obvious technique that has an easy clinical availability, is economical, and is practical, to compute the intra-operative dosimetry. One approach that has the promise of a comprehensive solution, is to register the seeds that are distinctly visible in X-ray (with no visibility of the soft tissue anatomy) to the prostate tissue segmented from TRUS (with marginal visibility of the seeds). This can potentially provide precise quantitative evaluation of the implant quality during the procedure, thereby allowing the physician to compensate for any error and achieve an optimal implant. The overall system concept for registration of ultrasound to fluoroscopy (RUF) is summarized in Fig. 1.

The implant procedure starts as usual. TRUS is used to guide each individual needle and a C-arm placed over the patient's abdomen. Upon implanting a batch of needles, the 3D locations of the implanted seeds are reconstructed using three X-ray images, and then superimposed over the spatially co-registered TRUS images. This allows for the implant plan to be optimized to account for discrepancies from the ideal dose distribution. The procedure can continue described above, with the next batch of needles in the cycle. After the last needle, a complete dosimetry check can be performed, providing a final opportunity to patch up any cold spots with additional seeds.

Quantitative use of fluoroscopy for implant dosimetry is currently not possible owing to a plethora of technical problems. As illustrated in Fig. 2, the main technical problems towards 3D C-arm reconstruction are pose-dependent $\mathrm{C}$-arm tracking, distortion correction and 
calibration; seed segmentation, matching and reconstruction; and finally the registration of the $\mathrm{C}$-arm to TRUS images. We develop tools that render this possible, making it available for safe, simple, and robust intra-operative 3D localization of brachytherapy sources with respect to the prostate. Furthermore, the $\mathrm{C}$-arm motion is limited to not more than $15^{\circ}$ around the AP-axis, due to collision hazards with the patient and operating table.

Significant efforts have been made toward computational fluoroscopy in general surgery (Yao et al., 2000; Hofstetter et al., 1999), developing various fundamental tools for 3D guidance. While several groups have published protocols (Nori et al., 1997; Nag et al., 1995; Wallner et al., 1991) and clinical outcomes favorably supporting C-arm fluoroscopy for intra-operative dosimetric analysis (Reed et al., 2005; French et al., 2005; Ravindran et al., 2004; Todor et al., 2003; Gong et al., 2002; Grado et al., 1998), this technique is yet to become a standard of care across hospitals. Most authors so far have concentrated on seed matching (Singh et al., 2007; Narayanan et al., 2004; Todor et al., 2002; Narayanan et al., 2002; Su et al., 2004; Tubic et al., 2001; Tutar et al., 2003; Altschuler and Kassaee, 1997; Lam et al., 2004; Murphy et al., 2005), for which in-vitro fluoroscopy experiments were carried out on well calibrated equipment. These devices are fully-encoded, calibrated, and distortion-corrected isocentric machines that provide an idealistic, virtually nonexistent representation of a $\mathrm{C}$-arm fluoroscope typically used in the brachytherapy suite. Here, we do not go into the specific details of each publication, but cluster them together to show the emerging pattern.

The biggest bottleneck towards impacting the standard of care has been the capability to use a typical non-isocentric $\mathrm{C}$-arm, present in most hospitals. Most $\mathrm{C}$-arms are non-isocentric, i.e. as the $\mathrm{C}$-arm rotates along any of its primary axes, it does not have a fixed axis of rotation. Modern isocentric $\mathrm{C}$-arms have the advantage that by just using the encoder reading, the pose of the $\mathrm{C}$-arm can be accurately computed. Non-isocentric $\mathrm{C}$-arms on the other hand, require some external tracking hardware for tracking. Furthermore, due to the poor manufacturing quality amongst non-isocentric ' $\mathrm{Cs}$ ', their calibration changes significantly from one pose to another. This makes their use for accurate 3D guidance extremely challenging. Though, fluoroscopic guidance in general surgery has developed methods to tackle these issues, those solutions have not extended easily and robustly for routine clinical use, in particularly for Brachytherapy.

We would discuss in detail in the following section each of the above problems. Here we just note that (Orio et al., 2007; Reed et al., 2005; French et al., 2005; Todor et al., 2003) have been the most important attempts to fuse X-ray fluoroscopy with TRUS. Important works as they are, they exhibited limited success. Seed reconstruction using three images using an isocentric C-arm has been achieved (Orio et al., 2007; Reed et al., 2005; Ravindran et al., 2004; Gong et al., 2002), and later registered to the TRUS images (Tutar et al., 2008) using seeds. The use of an isocentric C-arm (Todor et al., 2003; Todor et al., 2002) is usually the status-quo, though alternate approaches for $\mathrm{C}$-arm tracking that could extend on non-isocentric C-arms (Zhang et al., 2004) have also been proposed. However, we have not as of yet notice the reporting of any successful clinical system. A different approach is to not reconstruct the seeds from X-ray images (French et al., 2005), but instead merged x, y-axis information about the seeds from a single X-ray image, with the $\mathrm{z}$-axis information obtained 
from TRUS. Though this provides an inaccurate $\mathrm{X}$-ray reconstruction, it is the best they could do, since their clinical protocol did not allow for multiple X-ray images.

Altogether, a comprehensive and clinically reliable solutions for implant reconstruction from fluoroscopic data and spatial registration of fluoroscopy to TRUS, is currently not available. We report a new system that reconstructs 3D seed locations (visible in X-ray) and spatially registers them to the prostate (visible in TRUS). Preliminary conclusions on technical validation have been presented earlier (Jain et al., 2007), which have been further expanded here with quantitative clinical conclusions on edema characteristics. We discuss briefly the variety of technical issues in Section 2, following up with results from phantom experiments and a Phase-I clinical trials in Section 3. Our primary contribution to the state of the art is our ability to use any typical non-isocentric uncalibrated $\mathrm{C}$-arm present in most hospitals. We believe that the availability of this technology, followed up with large scale clinical studies and commercialization (ongoing), promises to lead to a paradigm shift in the standard of care for image-guided prostate brachytherapy.

\section{Methods and materials}

The system is designed to integrate easily with commercial brachytherapy installations. We employ a regular clinical brachytherapy setup, without alteration, including a treatment planning workstation and stabilizer/stepper (Interplant ${ }^{\circledR}$, CMS, St Louis), TRUS (B\&K Medical Pro Focus) and a C-arm (GE OEC 9600/9800). The C-arm is interfaced with a laptop through an NTSC video line and frame grabber, making the image capture independent of the $\mathrm{C}$-arm model.

\subsection{Workflow}

The clinical workflow (Fig. 3) is identical to the standard procedure until the clinician decides to run a reconstruction and optimization. A set of C-arm images are collected with $a$ separation as wide as clinically possible (usually $10^{\circ}$ around $\mathrm{AP}$-axis) and synchronously transferred to the laptop. After processing the images, the seeds are reconstructed and their 3D locations exported to the Interplant ${ }^{\circledR}$ system. The physician uses standard Interplant ${ }^{\circledR}$ tools to analyze, optimize and modify the remainder of the plan. The procedure concludes when the exit dosimetry shows no cold spots (under-radiated locations).

Numerous technical obstacles have to be overcome to realize C-arm based intra-operative dosimetry: (a) pose estimation of C-arm images; (b) C-arm calibration; (c) image distortion correction; (d) seed segmentation; (e) seed matching and reconstruction; (f) registration of $\mathrm{C}$-arm to TRUS; (g) dosimetry analysis; and finally (h) implant optimization. We have developed a system that overcomes these limitations in providing quantitative intraoperative dosimetry. In what follows, we will describe briefly each component of the system, skipping the detailed mathematical framework.

\subsection{Pose estimation}

The most critical component of a clinically usable solution for 3D fluoroscopic guidance is $\mathrm{C}$-arm pose estimation. $\mathrm{C}$-arms available in most hospitals do not have encoded rotational joints, making the amount of $\mathrm{C}$-arm motion unavailable. $\mathrm{C}$-arm tracking using auxiliary 
trackers is expensive, inaccurate in the presence of metal (EM tracking) or intrudes in the operating room (optical tracking). There has been some prior work on fiducial based tracking, wherein a fiducial is introduced in the X-ray FOV and its projection in the image encodes the 6 DOF pose of the $\mathrm{C}$-arm. The most significant problem in contemporary designs is that though sufficiently accurate, they are too bulky to be easily used in a clinical setting. To improve clinical usability, the size of the fiducial can be decreased, usually at a significant expense of the accuracy.

We proposed a new fluoroscope tracking fiducial design, FTRAC, that uses an ellipse (Jain et al., 2005; Jain et al., 2006). The fiducial is illustrated in Fig. 4. The ellipse makes pose recovery accurate by (a) always projecting as an ellipse; (b) allowing an accurate segmentation; and (c) providing closed form Jacobian formulations for fast optimization. The FTRAC design has salient features, including small dimensions $(3 \times 3 \times 5 \mathrm{~cm})$, no special proximity requirements to the anatomy, and is relatively inexpensive. In particular, the small size makes it easier to be always in the FOV and to be robust to image distortion. Extensive phantom experiments indicated a mean tracking accuracy on distorted C-arms of $0.56 \mathrm{~mm}$ in translation and $0.19^{\circ}$ in rotation, an accuracy comparable to expensive external trackers.

\subsection{C-arm source calibration and image distortion}

Since both C-arm calibration and distortion are pose-dependent, contemporary fluoroscopy guidance systems calibrate/distortion-correct at each imaging pose. This is done using a cumbersome calibration-fixture, which is a significant liability. Our approach is a complete departure. Using a mathematical and experimental framework, we demonstrated that calibration is not critical for prostate seed reconstruction; i.e. just an approximate preoperative calibration suffices. The central intuition is that object reconstruction using a miscalibrated C-arm changes only the absolute positions of the objects, but not their relative ones (Fig. 5). Additionally, statistical analysis of the distortion on a GE OEC 9600, inside a $15^{\circ}$ limited workspace revealed that just a single pre-operative correction at the AP-axis can reduce the average distortion in the image from $3.31 \mathrm{~mm}$ to $0.51 \mathrm{~mm}$. These errors are expected to be similar for most modern C-arms, with a more involved discussion available in (Jain et al., 2007). This residual amount of distortion in the X-ray image is acceptable for accurate 3D reconstruction, especially when used with image based C-arm tracking.

\subsection{Seed segmentation}

Seed segmentation in C-arm video images is usually hindered by poor image resolution. We implemented an automated seed segmentation algorithm that employs the morphological top-hat transform to perform the basic seed segmentation, followed by thresholding, region labeling, and finally a statistical classification into two classes - single seeds and clusters containing overlapping seeds. The algorithm is similar to one proposed earlier (Todor et al., 2003), with minor additions to improve classification. Using some simple ellipse partitioning techniques, these clustered seeds are finally broken down into single seeds. The algorithm was tested on clinical images and found to be sufficiently accurate. Out of total 763 seeds from 10 patient images of Palladium implants, 758 seeds were correctly identified; only two seeds clusters were not correctly resolved and two false positives were 
added. The segmentation is verified on the screen to allow for a manual bypass by the surgeon (Figs. 6 and 7).

\subsection{Seed correspondence and reconstruction}

The 3D coordinates of the implanted seeds can now be triangulated by resolving the correspondence of seeds in the multiple X-ray images. As explained in (Kon et al., 2006) we formalized the seed correspondence as a combinatorial optimization problem. As seen in Fig. 8, a network is created where any flow represents a matching, the desired solution being the flow with minimum cost, computed using the cycle-canceling algorithm. Our formulation has many preferred features: (a) exact solutions; (b) claims on the problem complexity; and (c) optimality considerations. We showed that a polynomial-time perfect solution is not achievable and proposed a practical solution (MARSHAL) that runs practically in $\mathrm{O}\left(\mathrm{N}^{3}\right)$ using any number of images, where $\mathrm{N}$ is the number of inserted seeds. In comparison, previous solutions have predominantly been heuristic explorations of the large search space. In addition, the framework robustly resolves all the seeds that are hidden in the images (as many as $7 \%$ can be hidden due to the high seed density). A simple extension to the network at strategic nodes, allowing a flow of two units instead of 1 , enables MARSHAL to also automatically recover hidden seeds. MARSHAL typically reconstructs $99.8 \%$ of the seeds and runs in under $5 \mathrm{~s}$ in MATLAB, exhibiting a significantly higher than the required minimum-detection-rate of $95 \%$ that has been suggested (Su et al., 2005).

\subsection{Registration of C-arm to TRUS}

The 3D seed coordinates are reconstructed with respect to the FTRAC and need to be registered to the prostate boundaries visible in the TRUS images. This is a difficult problem, owing to the lack of physical access to the patient-anatomy and the inherently complementary natures of the two modalities. X-rays predominantly images hard tissue, while Ultrasound looks at soft tissue boundaries. In a quick survey of X-ray to TRUS registration, various groups have attempted to use catheters, gold marker seeds, needles or the inserted radioactive seeds as markers. Unfortunately, implanted markers in Ultrasound are very hard to differentiate from various other objects like inserted seeds and needle tracts. Moreover, they are also susceptible to intra-operative tissue expansion, making the registration unreliable. Alternately, radio-opaque beads were attached to the probe and have been used for registration, requiring a permanent alteration to the probe, which for many practitioners is not desirable. Altogether, a comprehensive and clinically reliable solution for spatial registration of fluoroscopy and TRUS images is currently not known.

In commercial brachytherapy systems the needle insertion template is already pre-registered to TRUS probe as per the FDA approved clinical protocol. Hence, we propose to mount the FTRAC fiducial on top of this template in a known rigid position, using a precisionmachined mechanical connector. The arrangement is illustrated in Fig. 9. Thus a simple application of the various known frame transformations, registers the 3D seeds (FTRAC) to the prostate (TRUS). 


\subsection{System implementation}

We have integrated the previously discussed functions into a complete MATLAB program with a graphical user interface. Screen-captures from the program are shown in Fig. 11. The package runs on an ordinary laptop that sends reconstructed seed positions to Interplant ${ }^{\circledR}$ system. In order to not require a new FDA approval, we maintain the integrity of the FDAapproved Interplant ${ }^{\circledR}$ system by not modifying the commercial software. We instead use a text file to export the 3D seed locations.

\subsection{Dosimetry analysis and implant optimization}

The seed locations (in template coordinates) are exported to the Interplant ${ }^{\circledR}$ system. A software patch added to the Interplant ${ }^{\circledR}$ removes the already implanted seeds from the original plan, thereby producing a "residual implant plan". The total dose is calculated by combining the current seed locations and those in the residual implant plan. The physician at this stage, modifies the residual plan to avoid any potential 'hot spots' (prostate tissue receiving significantly more than prescribed radiation dose), and most importantly, fills in any observed cold spots (under-dosed prostate tissue receiving less than prescribed radiation). This process can be repeated any number of times during the surgery, achieving a more homogenous seed distribution.

\subsection{Clinical workflow}

The procedure flows in two main branches as shown in Fig. 12. In addition to the FDA approved TRUS-template calibration, the pre-operative phase now also includes the $\mathrm{C}$-arm calibration. Intraoperatively, the procedure proceeds without any alterations. At any point, the physician can decide to incorporate the current 3D seed locations, in which an X-ray reconstruction is carried out. The TRUS probe is retracted to prevent the transducer from blocking the C-arm's field of view. With moving the C-arm over the target area, 3-4 X-ray images are collected. Each image provides a view of the prostate (with the implanted seeds) and the FTRAC fiducial. One has to take care that both the seeds and the fiducial are visible in the images. In our experience, this has not been a critical problem when we position the AP image to have both visible in the center of the image. The fact that the allowed motion range is only about $15^{\circ}$ is of help here. These images are then automatically captured by the computer using a framegrabber. The intra-operative workflow continues with extensive image analysis, as described so far. Each C-arm image is automatically corrected for distortion. Next, the segmentation algorithm segments the motifs of the FTRAC fiducial and recovers the relative poses of the $\mathrm{C}$-arm images, followed by the segmentation of all visible seeds in the images. MARSHAL then matches all the segmented seeds and reconstructs their 3D locations, while also recovering the seeds hidden in one or more images. Finally, we apply the predetermined coordinate transformation to the seed cloud to obtain the position of the seeds in template coordinates and send them to the Interplant ${ }^{\circledR}$, where the dose coverage relative to the relevant anatomy is analyzed and the remainder of the implant plan optimized. At this point, the clinical workflow reverts back to the default standard of care protocol.

In preparation of the validation of the proposed system, we introduce the terms absolute and relative reconstruction errors. Using $\mathrm{X}$-ray images, the seeds are reconstructed with respect to the FTRAC frame. In experiments where the ground truth location of the seeds with 
respect to the FTRAC is known, the comparative analysis of the seed locations is called absolute accuracy. Sometimes (e.g. in patients), the true seed locations with respect to the FTRAC are not available and the reconstruction can only be compared to the seeds extracted from post-operative data (using a rigid point-cloud to point-cloud transform), in which case the evaluation is called relative accuracy.

\section{Phantom experiments and results}

We have extensively tested the system and its components in various phantoms and in a Phase-1 clinical trial.

\subsection{Solid seed phantom}

An acetol (Delrin) phantom consisting of ten slabs (5 mm each) was fabricated (Fig. 13). This phantom provides a multitude of implants with sub-mm ground truth accuracy. The fiducial was rigidly attached to the phantom in a known way, establishing the accurate ground truth 3D location of each seed. Realistic prostate implants (1.56 seeds/cc, 40-100 seeds) were imaged within a $30^{\circ}$ cone around the AP-axis. The true correspondence was manually established by using the 3D locations, known from the precise fabrication. Averaged results indicate that we correctly match $98.5 \%$ and $99.8 \%$ of the seeds using 3 and 4 images (100 and 75 total trials) respectively. The mean 3D absolute reconstruction accuracy was $0.66 \mathrm{~mm}$ (STD $0.29 \mathrm{~mm}$ ), while the relative accuracy was $0.35 \mathrm{~mm}$. The maximum reconstruction error (among few mismatched seeds) was $1.96 \mathrm{~mm}$ and $1.58 \mathrm{~mm}$ for three and four images respectively. The reconstruction accuracy of correctly matched seeds was always very 'tight', varying only due to segmentation accuracy (1-2 pixels) - it always was always within the $1 \mathrm{~mm}$ limit. Furthermore, using 4 images yielded only one poorly mismatched seed from the 75 datasets, suggesting the use of 4 images for better clinical guidance.

\subsection{Soft training phantoms}

As annotated in Fig. 14, we fully seeded three standard prostate brachytherapy phantoms with realistic implant plans (45, 49, 87 seeds). Seed locations reconstructed from fluoro using realistic (maximum available clinically) image separation (about $15^{\circ}$ ) were compared to their corresponding ground truth locations segmented manually in CT (1 $\mathrm{mm}$ slice thickness). Additionally, the 45 and 87-seed phantoms were rigidly attached to the FTRAC, providing the absolute ground truth (from CT). The 49-seed phantom was used to conduct a full scale practice-surgery, in which case the $3 \mathrm{D}$ reconstruction could be compared only to the seed cloud from post-op CT (without FTRAC), providing just relative accuracy.

The performance of the system on the soft tissue training phantoms is tabulated in Table 1. The absolute reconstruction errors for the 45, 87-seed phantoms were $1.64 \mathrm{~mm}$ and $0.95 \mathrm{~mm}$ (STD $0.17 \mathrm{~mm}$ ), while the relative reconstruction errors for the 45, 49, 87-seed phantoms were $0.22 \mathrm{~mm}, 0.29 \mathrm{~mm}, 0.20 \mathrm{~mm}$ (STD $0.13 \mathrm{~mm}$ ). A mean translation shift of $1.32 \mathrm{~mm}$ was observed in the $3 \mathrm{D}$ reconstructions, predominantly due to the limited C-arm workspace (solid-phantom experiments with $30^{\circ}$ motion have $0.65 \mathrm{~mm}$ accuracy). It was observed that the shift was mostly random and not in any particular direction. In fact, it was noticed that if 
different combinations of the X-ray images were used, it would result in different directions for the overall shift. This suggests that the shift is most probably due to the small amounts of error (sub-mm, sub-degree) in the pose estimation part of the system. Note that our reconstruction accuracy is better than the CT resolution (as evident from the solid seed phantom experiments). The accuracy is more than sufficient for brachytherapy, especially since a small shift does not hamper the ability to detect the presence of any cold spots.

\subsection{Patients}

A total of 18 batches of reconstructions were carried out on six patients with 2-4 batches/ patient, as and when a need for dose evaluation was felt by the clinician. The number of seeds in each batch varied from $22-84$. Since the seeds migrate significantly by the time a post-operative $\mathrm{CT}$ is taken, there is no easy method for knowing the true $3 \mathrm{D}$ seed locations (ground truth) in real patients. Hence, for each reconstruction 4-6 additional X-ray images were taken. The intra-operatively reconstructed 3D seed locations were projected on these additional images and compared to their corresponding segmented 2D locations (postoperatively). The results from a total of 99 such projections from 18 reconstructions are tabulated in Table 2 .

The results indicate a 2D mean error of $1.57 \mathrm{~mm}$ (STD $0.34 \mathrm{~mm}$, max $2.50 \mathrm{~mm}$, min 0.83 $\mathrm{mm})$. This indicates a sub-mm accuracy in 3D reconstruction, since the errors get magnified under a perspective transform when projected from 3D to a $2 \mathrm{D}$ image. Though the magnification factor varies for each image depending on the relative depth of the seeds with respect to the $\mathrm{C}$-arm focal length, it ranges approximately from 1.5 to 2 . This indicates an average absolute 3D reconstruction accuracy of $0.8-1.0 \mathrm{~mm}$, which is similar to the results obtained from the phantom experiments. Further note that the average minimum error is $0.83 \mathrm{~mm}$, indicating that there is a small consistent shift/bias in the reconstruction. This collaborates completely with the observations that were made earlier. In only one case (patient-2 84-seeds), we see a maximum error more than $4 \mathrm{~mm}$. However, in these cases we also observe that the over all deviation is higher too, indicating that these datasets might have a larger 3D shift/bias. In any case, we do not see the maximum error go many deviations away from the mean error. A $2.5 \mathrm{~mm}$ error in 3D is expected to project as a $5 \mathrm{~mm}$ error at least in some of the X-ray images, which we have not observed. This indicates that we had successful intra-operative reconstructions in the Phase-I patient trials.

\subsection{Registration accuracy}

To measure the accuracy of the fiducial-to-template registration, three batches of five straight needles each were inserted randomly at known depths ( $Z$-axis) into known template holes ( $X, Y$-axis). Their reconstructed tip locations with respect to the FTRAC coordinateframe were transformed to the template coordinate-frame using our rigid registration transform, which were then compared to their true measured locations in template coordinates. The limited-angle image-capture protocol was kept similar to that used in the operating room. Both absolute and relative results are tabulated in Table 3. The average absolute error (reconstruction together with registration) was $1.03 \mathrm{~mm}$ (STD $0.18 \mathrm{~mm}$ ), while the average relative error was $0.36 \mathrm{~mm}$ (STD $0.21 \mathrm{~mm}$ ), with an average translation shift of $0.97 \mathrm{~mm}$. Note that the performance of the system in these experiments is similar to

Med Image Anal. Author manuscript; available in PMC 2015 December 30. 
the performance of the system in the other experiments presented so far, strengthening the validity of our conclusions.

\subsection{System accuracy}

To measure the full system error, 5 needles (tips) were inserted into a prostate brachytherapy training phantom, reconstructed in $3 \mathrm{D}$ and exported to the Interplant ${ }^{\circledR}$ software. The needle tips were also segmented manually using TRUS images. The sagittal images were used for measuring the depth ( $Z$-axis) of the needles and the transverse images were used to measure the planar positions ( $X, Y$-axis), providing ground truth from Ultrasound. The mean absolute error for the five needle tips was $4 \mathrm{~mm}$ (STD $0.53 \mathrm{~mm}$ ), with a translation shift of $3.94 \mathrm{~mm}$. In comparison, the relative accuracy for the complete system was $0.83 \mathrm{~mm}$ (STD $0.18 \mathrm{~mm}$ ).

The shift can mainly be attributed to (i) an error in the Template-TRUS pre-calibration done as part of current clinical practice, resulting in a shift $(\sim 3 \mathrm{~mm})$; and (ii) a random bias in the $3 \mathrm{D}$ reconstruction due to limited image separation $(\sim 1 \mathrm{~mm})$. Nevertheless, we removed this shift in the clinical cases by applying a translation-offset to the reconstructed X-ray seed coordinates. Owing to the small size of the prostate, the rotation offset was always found to be minimal and could be ignored. The resultant translation-offset was intra-operatively estimated by comparing the centroid of the reconstructed seeds with that of the planned seed locations, and by aligning the two together. Note that the centroid is a first-order statistic and robust to any spatially symmetric noise/displacement model. Though a heuristic, it provided excellent qualitative results according to the surgeon, who read the visual cues at the reconstructed seed locations in TRUS images. Based on the experiments so far and the surgeon's feedback, the overall accuracy of the system is expected to be $1-2 \mathrm{~mm}$ during clinical use.

\subsection{Phase-I clinical trial}

We have treated a cohort of six patients, as part of a DoD Phase-I clinical trial to test the efficacy and safety of the system (the Phase-II trial is currently open for enrollment). Annotated photographs of the clinical setup are shown in Fig. 10 (c) and Fig. 14(b). Intraoperative dosimetry was performed (i) halfway during the surgery; (ii) at the end of the surgery; and (iii) after all the additional seeds were inserted. The current protocol adds about $15 \mathrm{~min}$ for each reconstruction, including the capture of 5 extra X-ray images for research validation, image processing, 3D seed reconstruction, and dosimetry optimization. In regular everyday clinical practice, we anticipate the need for only a single exitdosimetry reconstruction, increasing the operating time only by about $10 \mathrm{~min}$. In all the patients the final dosimetry detected cold spots (Fig. 15). The clinician grew quickly to trust the system in detecting cold spots, and instead minimized potential hot spots during the surgery. The medical team found the intra-operative visualization of under-dosed regions valuable, inserting an additional 4.17 seeds on an average to make the $100 \%$ prescribed isodose contour cover the prostate. All patients were released from the operating room with satisfactory outcomes.

The intra-operative detection and visualization of edema was also made possible by the proposed system, as shown in Fig. 16. Edema is the swelling of the prostate tissue due to the 
repeated stress from the punctures created by needle-insertions and the high radioactivity from the dropped seeds. It is the single-most important source of bottleneck towards accurate dose delivery, arguably leaving behind cold spots. The seeds (and hence the prostate) show a clear tendency for outward migration from their drop positions (with maximums up to $16 \mathrm{~mm}$ ). In all the patients, by the end of the procedure, it was found that the apex of the prostate (surgeon-end) was under-dosed. The system is unique, in that it is the first of a kind that can intra-operatively compute the edema before the patient leaves the OR. Our current method estimates edema by comparing the final implanted location of the seeds with the planned and intermediate 3D positions. The system computed significant intra-operative edema in all the patients, with an average tissue expansion of $3.84 \mathrm{~mm}$ (STD $2.13 \mathrm{~mm}$ ). The current assumption for edema computation is that the 'cause' for errors is predominantly tissue expansion, as evident visually from the figure. Work is currently underway to mathematically construct a model to separate seed migration due to placement errors and that from tissue expansion. In the case that only a single exit dosimetry reconstruction is done, this model will be clearly able to quantify the edema.

A further comparison of the 3D final implant reconstructed using the X-ray images to Day-0 CT ( $2 \mathrm{~mm}$ slices) indicated an increased average post-operative edema of $4.39 \mathrm{~mm}$ (STD $2.60 \mathrm{~mm}$ ), indicating a further post-operative seed migration in a few hours after the patient leaves the operating room, as can be observed in Tables 4-6. However, post-operative seed migration is an inherent limitation of brachytherapy, the best solution being a calculated intra-operative over-dosing of the patient. This makes the detection of any intra-operative edema even more critical. Brachy-therapists have been traditionally accommodating for it by overdosing the patient. Though effective, it involves some skilled qualitative guesswork on behalf of the surgeon, potentially limiting cancer control in difficult cases. Note that, in such a scenario, a sub-mm accuracy in the placement of the seeds inside the prostate could have a lesser impact on long-term cancer control.

\section{Conclusion, shortcomings and future work}

A system for intra-operative brachytherapy seed monitoring has been presented. In precision-machined hard phantoms with 40-100 seeds, we correctly reconstructed $99.8 \%$ seeds with a mean 3D accuracy of $0.35 \mathrm{~mm}$. In soft tissue phantoms with $45-87$ seeds and clinically realistic $15^{\circ} \mathrm{C}$-arm motion, we correctly reconstructed $100 \%$ seeds with an accuracy of $0.24 \mathrm{~mm}$, sufficient for detection of any cold spots. 18 intra-operative reconstructions from a phase-I clinical study also show a correct 3D reconstruction with under $1 \mathrm{~mm}$ error. This accuracy is more than sufficient for accurate intra-operative monitoring of the plan, since the system was able to measure the extent of intra-operative seed migration, showing that it could be as high as $16.19 \mathrm{~mm}$ (mean $3.84 \mathrm{~mm}$ ). The proposed work was also successfully used in a Phase-I DoD clinical trial, showing usefulness and great potential.

The system (a) requires no significant hardware; (b) does not alter the current clinical workflow; (c) can be used with any C-arm; (d) integrates easily with any pre-existing brachytherapy installation; and (e) is economically feasible and scalable. There is some added radiation to the patient from the X-ray images, though insignificant when compared to 
that from the radioactive seeds. Though not critical, primary shortcomings include (a) 15 min additional surgery time; and (b) an error in the registration. Research is currently underway to remove these limitations, and to conduct large scale clinical studies using clinical indicators. After the completion of phase- 1 study, the proposed algorithms have been tightly integrated with the FDA approved commercial system in the past two years. As can be expected, this step though makes the system more clinically usable, has taken a significant effort, delaying the start of the phase-2 study. During the writing of this manuscript, a phase-2 study was recently opened for patient enrollment. Furthermore, the proposed system is the first of its kind that can intra-operatively detect seed migration using any non-isocentric $\mathrm{C}$-arm, achieving a significantly more homogeneous distribution and avoiding radiation hot/cold spots. All the above put together, promises to lead to a paradigm shift in the standard of care for image-guided prostate brachytherapy, considerably improving the patient quality of life.

\section{Acknowledgments}

This work has been supported by DoD PC050170 - Prostate Cancer Research Program (2005) pre-doctoral Traineeship Award, DoD PC050042, NIH SBIR 1R43CA099374 01, NSF EEC-9731478.

\section{References}

Altschuler M, Kassaee A. Automated matching of corresponding seed images of three simulator radiographs to allow 3d triangulation of implanted seeds. Phys. Med. Biol. 1997; 42(2):293-302. [PubMed: 9044413]

Blasko J, Mate T, Sylvester J, Grimm P, Cavanagh W. Brachytherapy for carcinoma of the prostate: techniques, patient selection, and clinical outcomes. Semin. Radiat. Oncol. 2002; 12(1):81-94. [PubMed: 11813153]

Brunet-Benkhoucha M, Lassalle S, Béliveau-Nadeau D, Reniers B, Donath D, Taussky D, Carrier JF. Clinical implementation of a digital tomosynthesis-based seed reconstruction algorithm for intraoperative postimplant dose evaluation in low dose rate prostate brachytherapy. Med. Phys. 2009; 36(11):5235-5544. [PubMed: 19994534]

Cooperberg M, et al. The contemporary management of prostate cancer in the united states: lessons from the cancer of the prostate strategic urologic research endeavor (capsure), a national disease registry. J. Urol. 2004; 171(4):1393-13401. [PubMed: 15017184]

D'Amico A, et al. Real-time magnetic resonance imaging-guided brachytherapy in the treatment of selected patients with clinically localized prostate cancer. J. Endourol. 2000; 14(4):367-370. [PubMed: 10910153]

Dumane, et al. Combined ultrasound-fluoroscopy approach to the intra-operative detection of seeds in prostate brachytherapy. ASTRO Annual Meeting; Poster. 2004.

Fichtinger G, et al. Robotically assisted prostate brachytherapy with transrectal ultrasound guidancephantom experiments. Brachytherapy. 2006; 5(1):14-26. [PubMed: 16563993]

French D, Morris J, Keyes M, Salcudean SE. Real-time dosimetry for prostate brachytherapy using trus and fluoroscopy. MICCAI. 2004; (2):983-991.

French, et al. Computing intra-operative dosimetry for prostate brachytherapy using trus and fluoroscopy. Acad. Rad. 2005; 12(10):1262-1272.

Gong L, Cho P, Han B, Wallner K, Sutlief S, Pathak S, Haynor D, Kim Y. Ultrasonography and fluoroscopic fusion for prostate brachytherapy dosimetry. Int. J. Radiat. Oncol. Biol. Phys. 2002; 54(5):1322-1330. [PubMed: 12459353]

Grado G, Larson T, Balch C, Grado M, Collins J, Kriegshauser J, Swanson G, Navickis R, Wilkes M. Actuarial disease-free survival after prostate cancer brachytherapy using interactive techniques 
with biplane ultrasound and fluoroscopic guidance. Int. J. Radiat. Oncol. Biol. Phys. 1998; 42(2): 289-298. [PubMed: 9788406]

Grimm P, Sylvester J. Advances in brachytherapy. Urology. 2004; 6(4):37-48.

Han B, Wallner K, Merrick G, Butler W, Sutlief S, Sylvester J. Prostate brachytherapy seed identification on post-implant trus images. Med. Phys. 2003; 30(5):898-900. [PubMed: 12772998]

Hofstetter R, Slomczykowski M, Sati M, Nolte L. Fluoroscopy as an imaging means for computerassisted surgical navigation. Comput. Aided Surg. 1999; 4(2):65-76. [PubMed: 10494136]

Holmes D, Davis B, Bruce C, Robb R. 3D visualization, analysis, and treatment of the prostate using trans-urethral ultrasound. Comput. Med. Imaging Graph. 2003; 27(5):339-349. [PubMed: 12821027]

Hurwitz M, et al. Three-dimensional real-time magnetic resonance-guided interstitial prostate brachytherapy optimizes radiation dose distribution resulting in a favorable acute side effect profile in patients with clinically localized prostate cancer. Tech. Urol. 2000; 6(2):89-94. [PubMed: 10798806]

Jain, A.; Deguet, A.; Iordachita, I.; Chintalapani, G.; Blevins, J.; Le, Y.; Armour, E.; Burdette, C.; Song, D.; Fichtinger, G. Intra-operative $3 \mathrm{~d}$ guidance in prostate brachytherapy using a nonisocentric c-arm. Medical Image Computing and Computer Assisted Interventions (MICCAI); 2007 Oct.

Jain, A.; Iordachita, I.; Fichtinger, G. A small size fluoroscope tracking fiducial. Annual Meeting, Computer Assisted Orthopedic Surgery; 2006 Jun.

Jain A, Mustufa T, Zhou Y, Burdette EC, Chirikjian G, Fichtinger G. A robust fluoroscope tracking (ftrac) fiducial. Med. Phys. 2005; 32(10):3185-3198. [PubMed: 16279072]

Jain, et al. C-arm calibration: is it really necessary?. SPIE Medical Imaging 2007, Presented at the Society of Photo-Optical Instrumentation Engineers (SPIE) Conference; 2007 Mar.

Jemal A, Siegel R, Ward E, Hao Y, Xu J, Thun M. Cancer statistics. CA Cancer J. Clin. 2004; 59(4)

Kaplan I, et al. Real-time computed tomography dosimetry during ultrasound-guided brachytherapy for prostate cancer. Brachytherapy. 2006; 5(3):147-151. [PubMed: 16864065]

Kon, R.; Jain, A.; Fichtinger, G. Hidden seed reconstruction from c-arm images in brachytherapy. IEEE ISBI; 2006 Apr. p. 526-29.

Koutrouvelis $\mathrm{P}$, et al. Salvage reimplantation in patient with local recurrent prostate carcinoma after brachytherapy with three dimensional computed tomography-guided permanent pararectal implant. Technol. Cancer Res. Treat. 2003; 2(4):339-344. [PubMed: 12892517]

Kumar P, Good R, Epstein B, Hussain M, Bartone F. Fluoroscopy guided transperineal percutaneous permanent 125iodine implantation of prostate cancer. Radiat. Med. 1985; 3(3):161-167. [PubMed: 3834521]

Lam S, Cho P, Marks R, Narayanan S. Three-dimensional seed reconstruction for prostate brachytherapy using hough trajectories. Phys. Med. Biol. 2004; 49(4):557-569. [PubMed: 15005165]

Mitri FG, Davis BJ, Greenleaf JF, Fatemi M. In vitro comparative study of vibro-acoustography versus pulse-echo ultrasound in imaging permanent prostate brachytherapy seeds. Ultrasonics. 2009b; 49(1):31-38. [PubMed: 18538365]

Molloy J, et al. Treatment planning considerations and quality assurance for ct-guided transischiorectal implantation of the prostate. Med. Phys. 1999; 26(9):1943-1951. [PubMed: 10505884]

Murphy M, Todor D. Demonstration of a forward iterative method to reconstruct brachytherapy seed configurations from x-ray projections. Phys. Med. Biol. 2005; 50:2715-2737. [PubMed: 15901965]

Nag S, Ciezki J, Cormack R, Doggett S, DeWyngaert K, Edmundson G, Stock R, Stone N, Yu Y, Zelefsky M. intra-operative planning and evaluation of permanent prostate brachytherapy: report of the american brachytherapy society. Int. J. Radiat. Oncol. Biol. Phys. 2001; 51(5):1422-1430. [PubMed: 11728703]

Nag S, Scaperoth D, Badalament R, Hall S, Burgers J. Transperineal palladium 103 prostate brachytherapy: analysis of morbidity and seed migration. Urology. 1995; 45(1):87-92. [PubMed: 7817484] 
Narayanan S, Cho P, Marks R. Fast cross-projection algorithm for reconstruction of seeds in prostate brachytherapy. Med. Phys. 2002; 29(7):1572-1579. [PubMed: 12148740]

Narayanan S, Cho P, Marks R. Three-dimensional seed reconstruction from an incomplete data set for prostate brachytherapy. Phys. Med. Biol. 2004; 49(15):3483-3494. [PubMed: 15379027]

Nori D, Moni J. Current issues in techniques of prostate brachytherapy. Semin. Surg. Oncol. 1997; 13(6):444-453. [PubMed: 9358592]

Orio PF III, Tutar IB, Narayanan S, Arthurs S, Cho PS, Kim Y, Merrick G, Wallner KE. intraoperative ultrasound-fluoroscopy fusion can enhance prostate brachytherapy quality. IJROBP. 2007; 69(1):302-307.

Peschel R, Colberg J. Surgery, brachytherapy, and external-beam radiotherapy for early prostate cancer. Lancet Oncol. 2003; 4(4):233-241. [PubMed: 12681267]

Polo A, Salembier C, Venselaar J, Hoskin P. Review of intra-operative imaging and planning techniques in permanent seed prostate brachytherapy. Radiother. Oncol. 2010 Jan.

Prestidge B, Prete J, Buchholz T, Friedland J, Stock R, Grimm P, Bice W. A survey of current clinical practice of permanent prostate brachytherapy in the united states. Int. J. Radiat. Oncol. Biol. Phys. 1998; 40(2):461-465. [PubMed: 9457836]

Ravindran, P.; Lewis, C.; Alphonsi, J.; Lindsay, P.; D’Souza, D. A c-arm based intra-operative dosimetry system for trans-rectal ultrasound guided prostate implant with $125 \mathrm{I}$ seeds. Proc. 14th Int. Conf. on the Use of Computers in Radiation Therapy; 2004.

Reed, et al. Intraoperative fluoroscopic dose assessment in prostate brachytherapy patients. Int. J. Radiat. Oncol. Biol. Phys. 2005; 63(1):301-307. [PubMed: 16111600]

Siewerdsen, J.; Daly, M.; Bahar, G.; Moseley, D.; Bootsma, G.; Chhabra, S.; Jaffray, D.; Irish, J. Multimode c-arm fluoroscopy, tomosynthesis, and conebeam ct for image-guided interventions: from proof of principle to patient protocols. SPIE Medical Imaging; Physics of Medical Imaging; 2007 Feb.

Singh V, Mukherjee L, Xu J, Hoffmann KR, Dinu PM, Podgorsak M. Brachytherapy seed localization using geometric and linear programming techniques. IEEE Transactions on Medical Imaging. 2007; 26(9):1291-1304. (special issue on Mathematical Methods in Biomedical Image Analysis). [PubMed: 17896600]

Su Y, Davis B, Herman M, RA R. Prostate brachytherapy seed localization by analysis of multiple projections: identifying and addressing the seed overlap problem. Med. Phys. 2004; 31(5):12771287. [PubMed: 15191320]

$\mathrm{Su}$, et al. Examination of dosimetry accuracy as a function of seed detection rate in permanent prostate brachytherapy. Med. Phys. 2005; 32(9):3049-3056. [PubMed: 16266119]

Talcott J, et al. Time course and predictors of symptoms after primary prostate cancer therapy. J. Clin. Oncol. 2003; 21:3979-3986. [PubMed: 14581420]

Taschereau R, et al. Seed misplacement and stabilizing needles in transperineal permanent prostate implants. Radiother. Oncol. 2000; 55(1):59-63. [PubMed: 10788689]

Todor D, Cohen G, Amols H, Zaider M. Operator-free, film-based 3d seed reconstruction in brachytherapy. Phys. Med. Biol. 2002; 47(12):2031-2048. [PubMed: 12118599]

Todor D, Zaider M, Cohen G, Worman M, Zelefsky M. intra-operative dynamic dosimetry for prostate implants. Phys. Med. Biol. 2003; 48(9):1153-1171. [PubMed: 12765329]

Tornes, A.; Eriksen, M. A new brachytherapy seed design for improved ultrasound visualization. IEEE Symposium on Ultrasonics; 2003 Oct. p. 1278-1283.

Tubic D, Zaccarin A, L B, Pouliot J. Automated seed detection and three-dimensional reconstruction. ii. Reconstruction of permanent prostate implants using simulated annealing. Med. Phys. 2001; 28(11):2272-2279. [PubMed: 11764032]

Tutar I, Managuli R, Shamdasani V, Cho P, Pathak S, Kim Y. Tomosynthesis-based localization of radioactive seeds in prostate brachytherapy. Med. Phys. 2003; 30(12):3135-3142. [PubMed: 14713080]

Tutar IB, Gong L, Narayanan S, Pathak SD, Cho PS, Wallner K, Kim Y. Seed-based transrectal ultrasound-fluoroscopy registration method for intra-operative dosimetry analysis of prostate brachytherapy. Med. Phys. 2008; 35(3):840-848. [PubMed: 18404921] 
Wallner K, Chiu-Tsao S, Roy J, Arterbery V, Whitmore W, Jain S, Minsky B, Russo P, Fuks Z. An improved method for computerized tomography-planned transperineal 125iodine prostate implants. J. Urol. 1991; 146(1):90-95. [PubMed: 1711591]

Wei Z, Wan G, Gardi L, Mills G, Downey D, Fenster A. Robot-assisted 3d-trus guided prostate brachytherapy: system integration and validation. Med. Phys. 2004; 31(3):539-548. [PubMed: 15070252]

Xue, J.; Gressen, E.; Jefferson, T. Feasibility of trus-based prostate post-implant dosimetry. AAPM Annual Meeting; Poster. 2004 Jul.

Yao J, Taylor RH, Goldberg RP, Kumar R, Bzostek A, Van VR, Kazanzides P, Gueziec A. A c-arm fluoroscopy-guided progressive cut refinement strategy using a surgical robot. Comput. Aided Surg. 2000; 5(6):373-390. [PubMed: 11295851]

Zhang M, Zaider M, Worman M, Cohen G. On the question of 3d seed reconstruction in prostate brachytherapy: the determination of x-ray source and film locations. Phys. Med. Biol. 2004; 49(19):N335-N345. [PubMed: 15552426] 


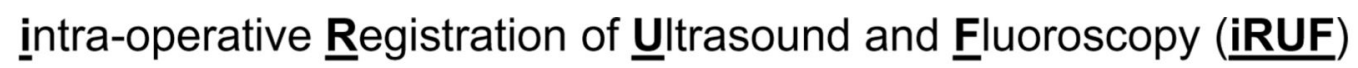

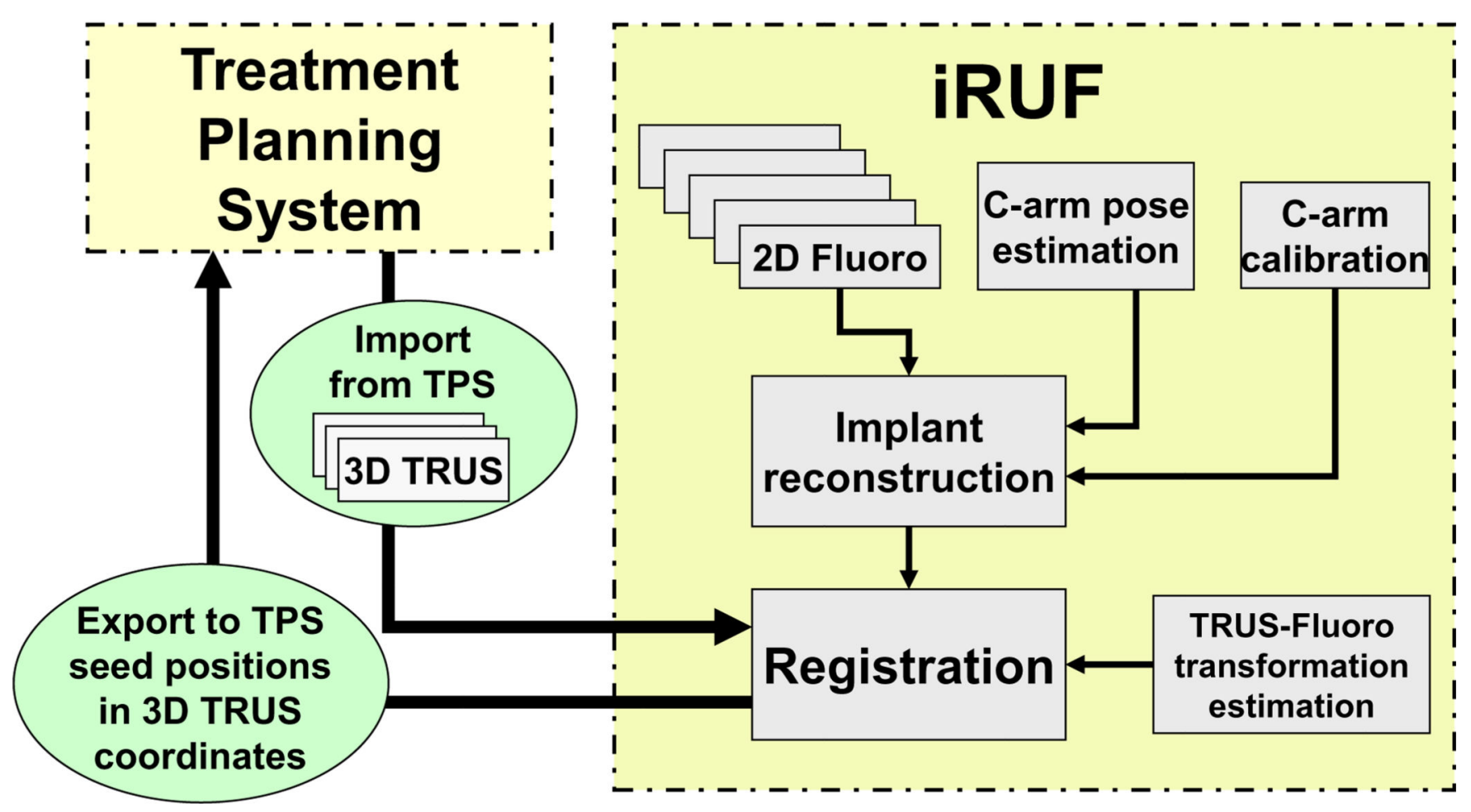

Fig. 1.

Overall system concept for TRUS and C-arm fusion. 


\section{C-arm Tracking}
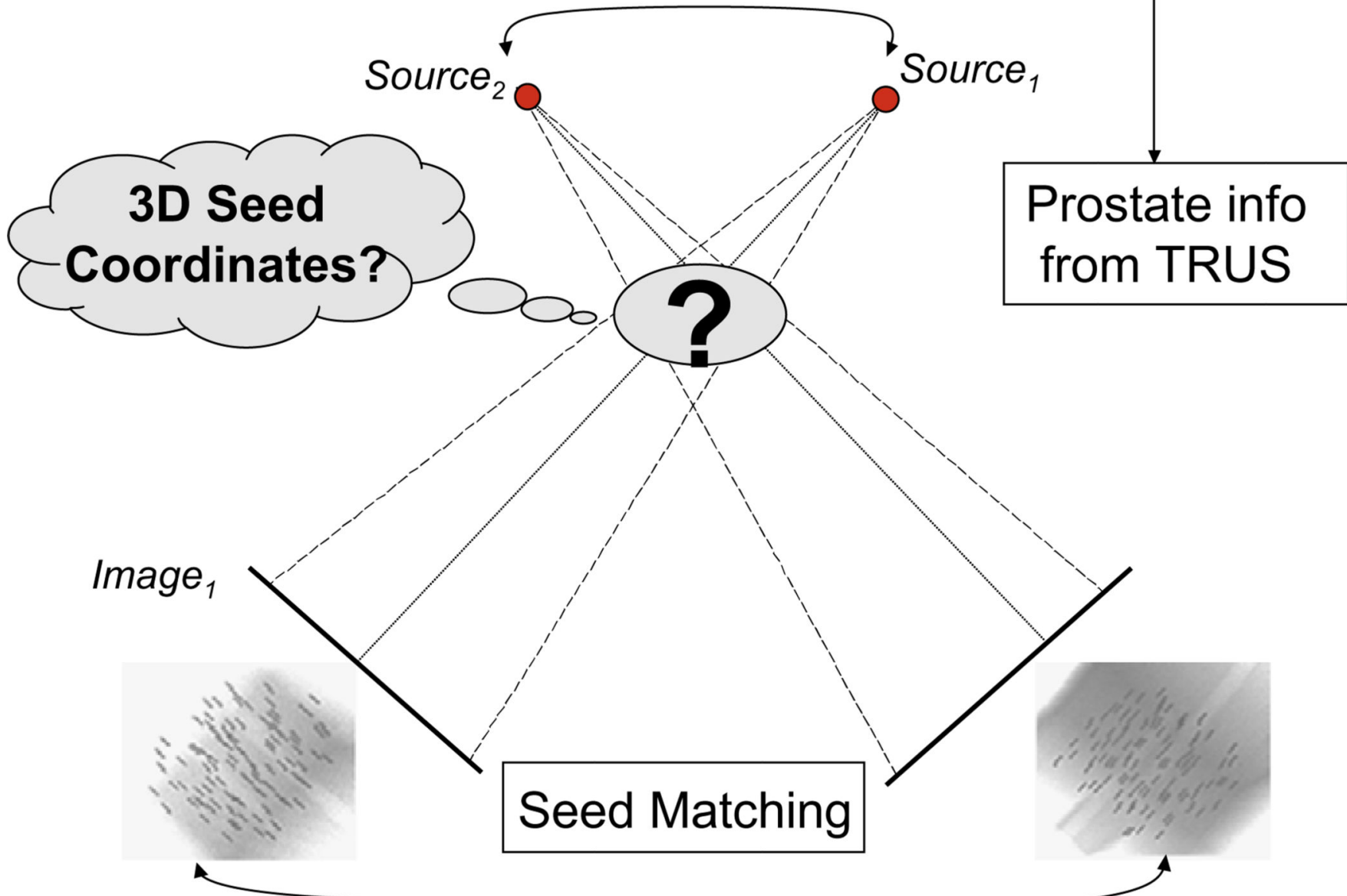

Fig. 2.

The main technical challenges towards intra-operative 3D seed reconstruction using C-arms. 


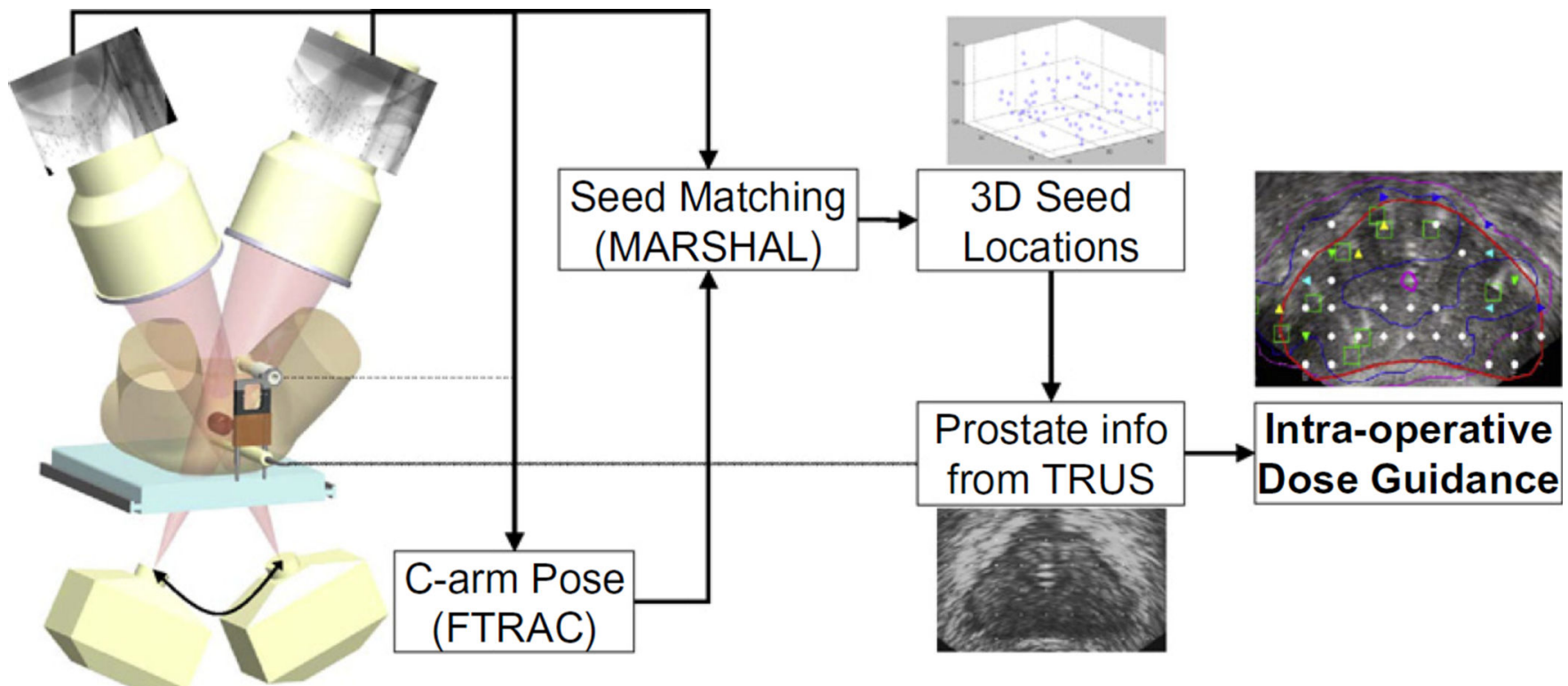

Fig. 3.

Overview of the proposed solution. The FTRAC fiducial tracks C-arms, and also registers TRUS to C-arm images, making quantitative brachytherapy possible. 


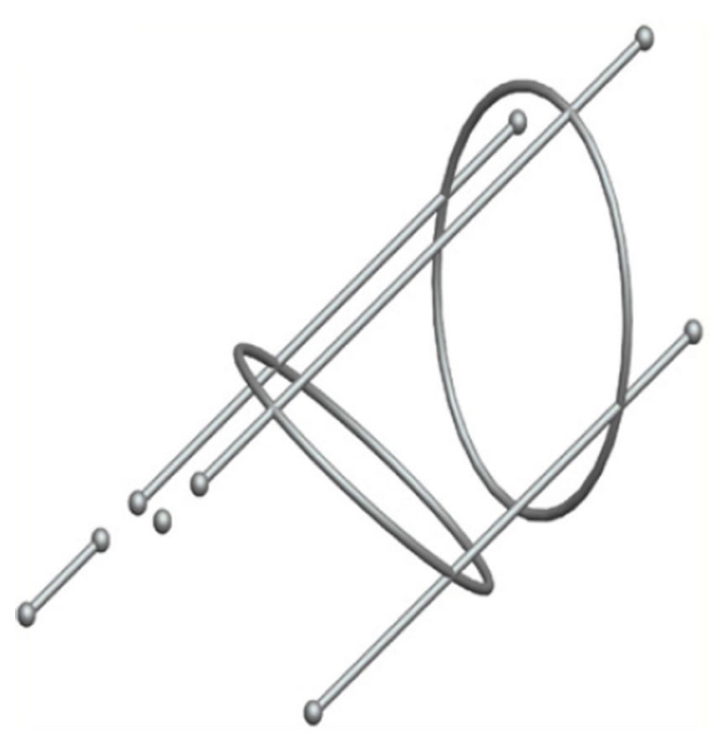

(a)

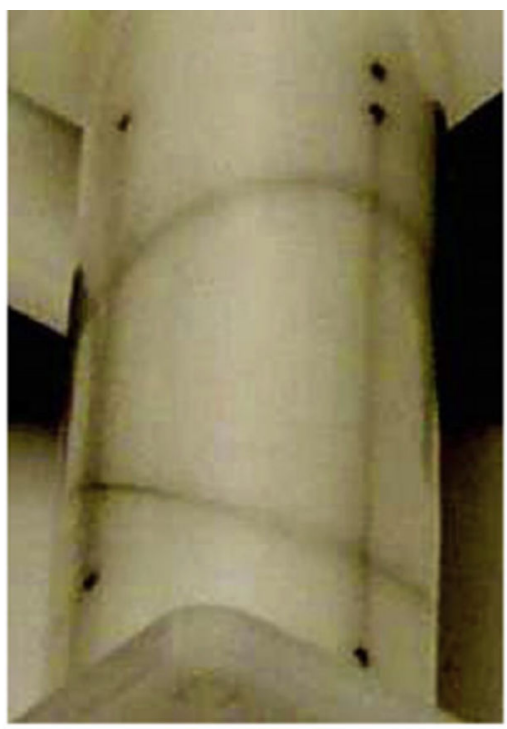

(b)

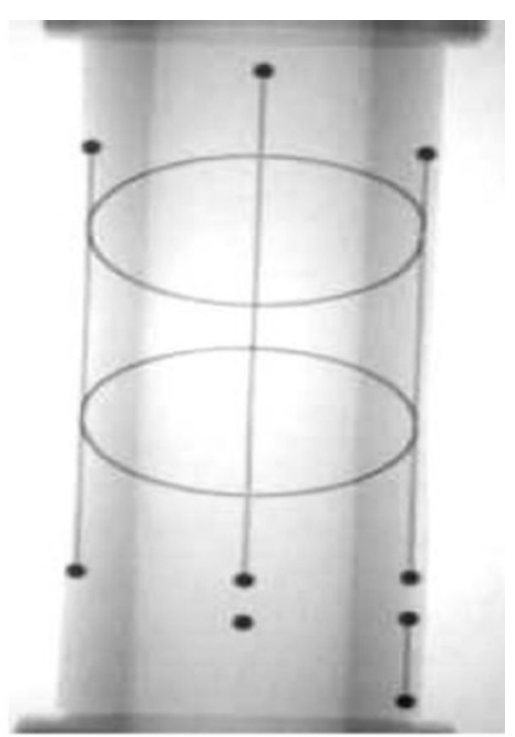

(c)

Fig. 4.

Images of the FTRAC fiducial (a) wire model; (b) photograph; (c) X-ray image. 


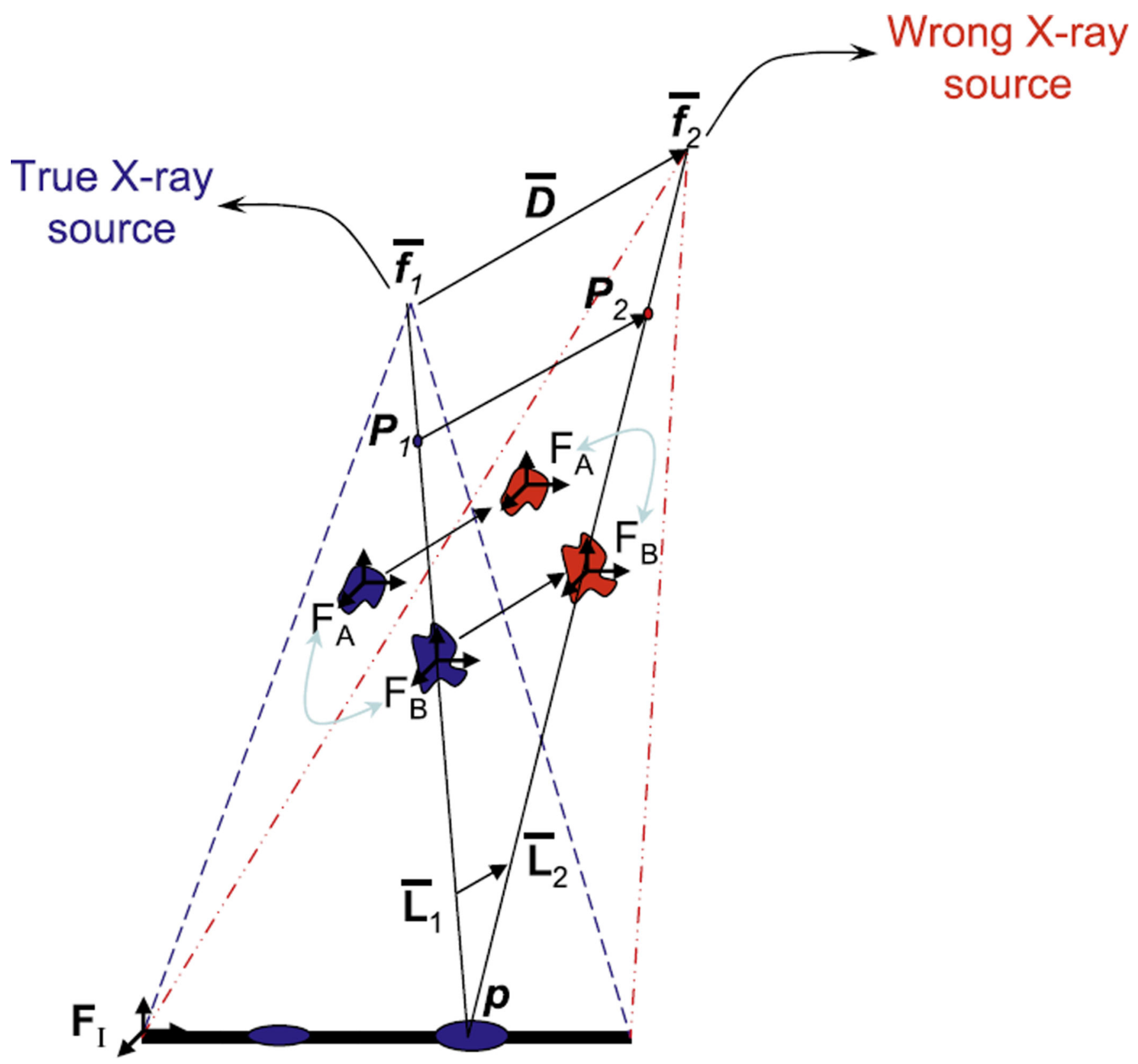

Fig. 5.

Mis-calibration conserves relative reconstruction between objects A and B (e.g. seeds). 

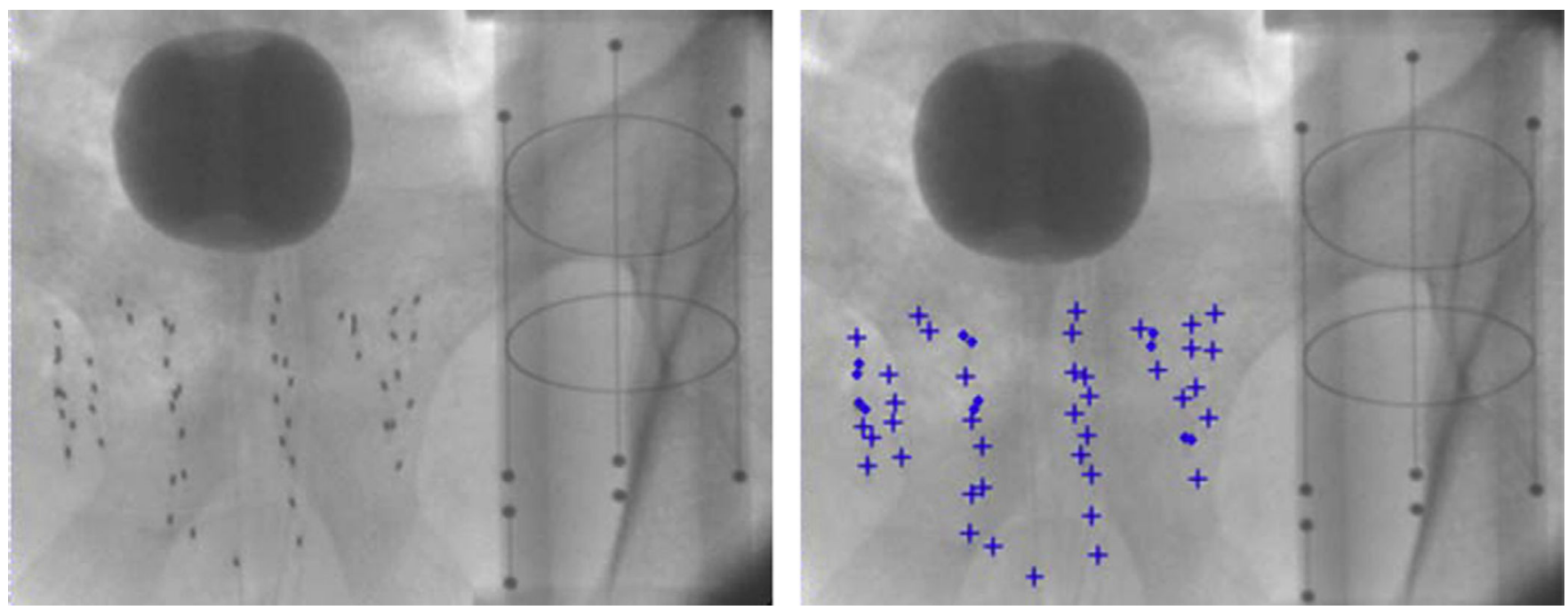

Fig. 6.

X-ray image before segmentation (left). There is also the FTRAC in the image. The image after segmentation (right); the blue ' + ' symbol represent individual seeds and the blue '.' indicates a seed that is a part of a multiple seed cluster. 


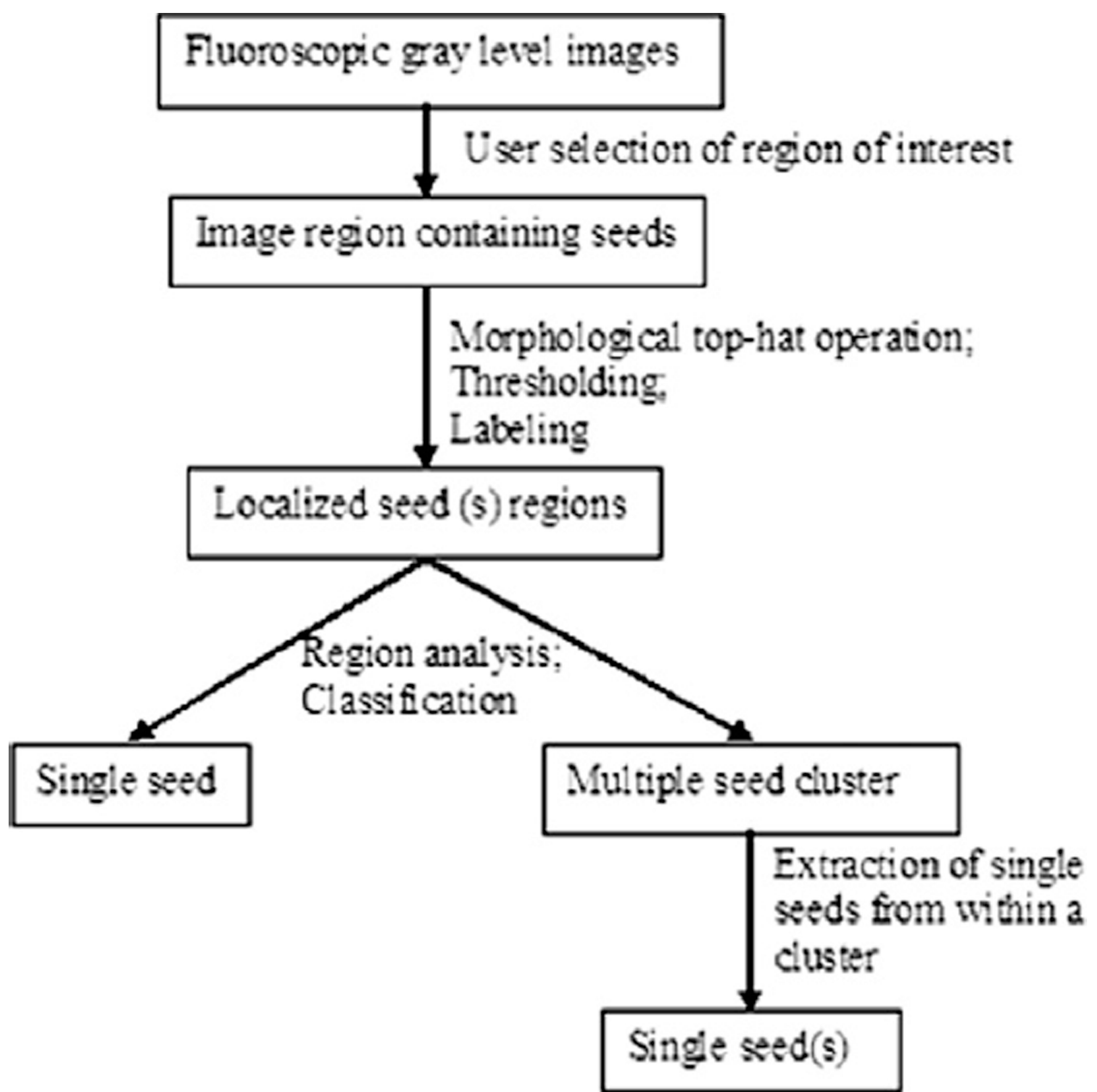

Fig. 7.

The flowchart of segmentation algorithm. 


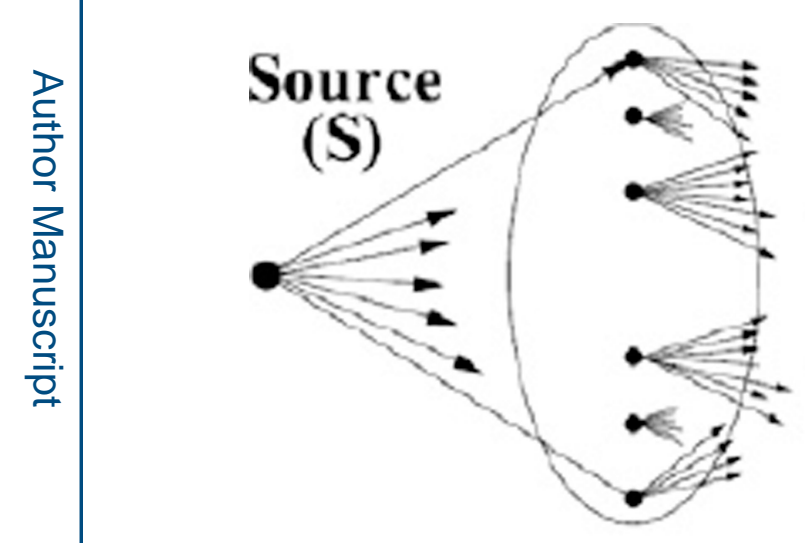

Set A (Image 1)

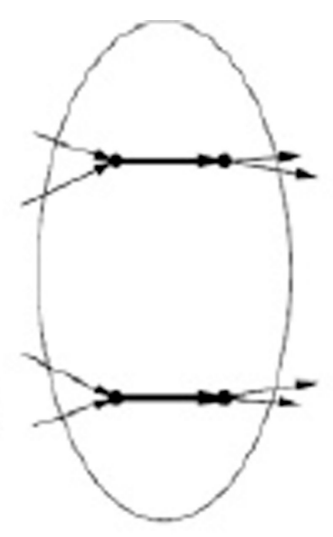

Set B

(Image2)

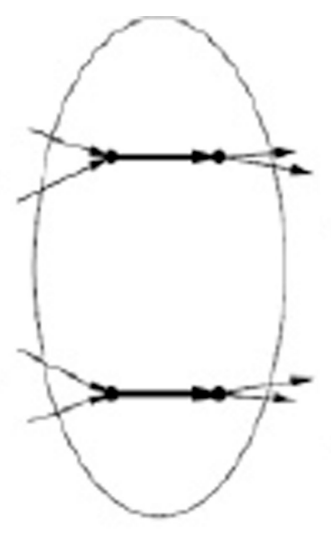

Set C

(Image3)

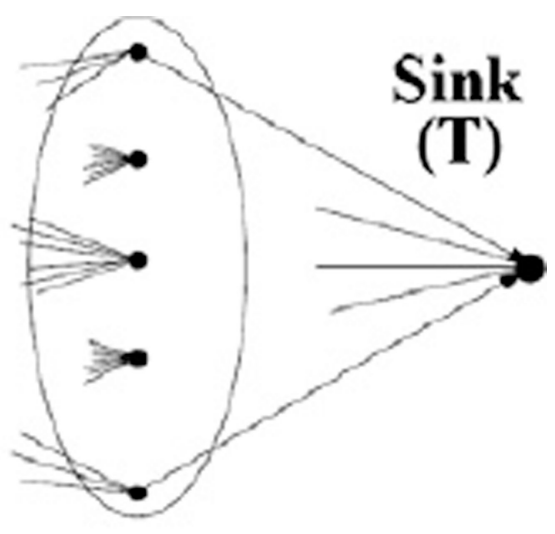

Set A

(Image1)

Fig. 8.

The network flow formulation used to solve the hidden-seed correspondence problem. 


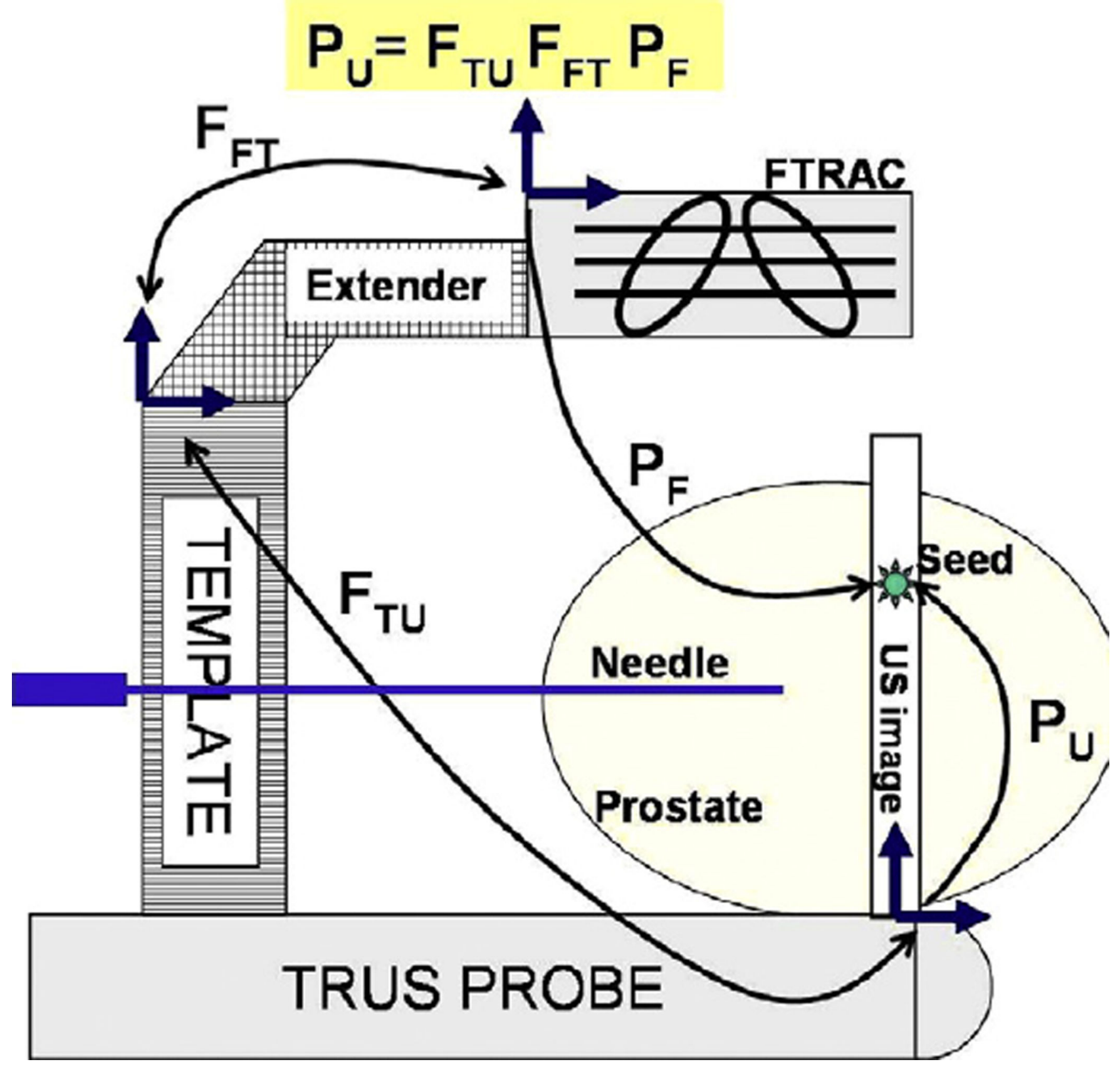

Fig. 9.

The frame transformations between the FTRAC and TRUS. The FTRAC fiducial and the needle insertion template can be pre-calibrated using a rigid mount. 


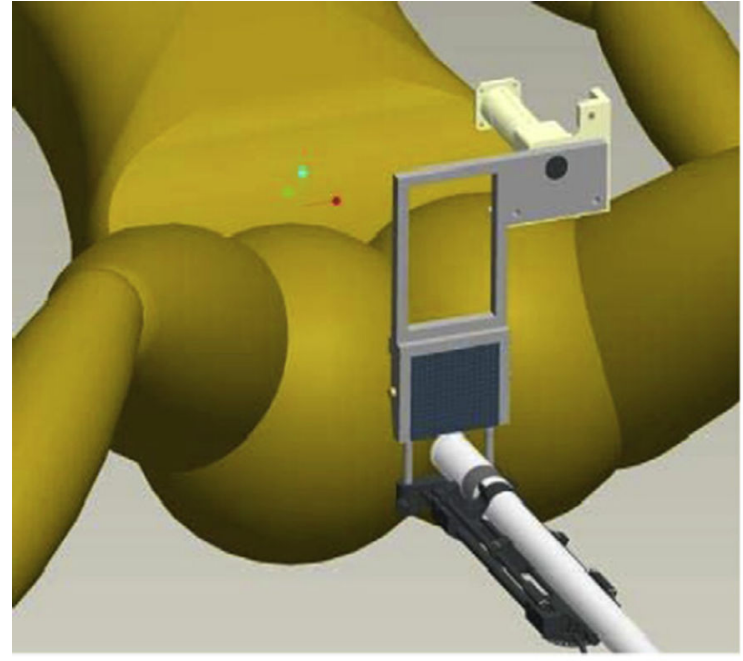

(a)

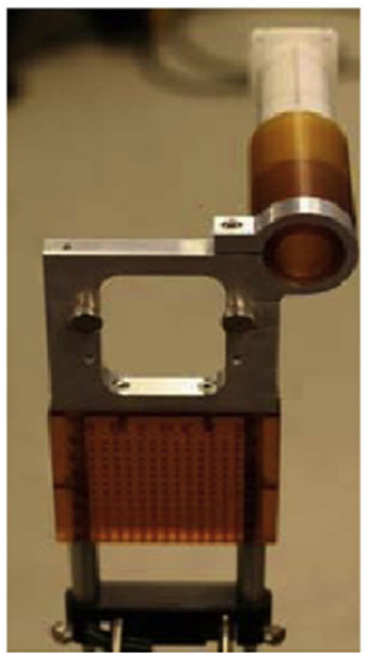

(b)

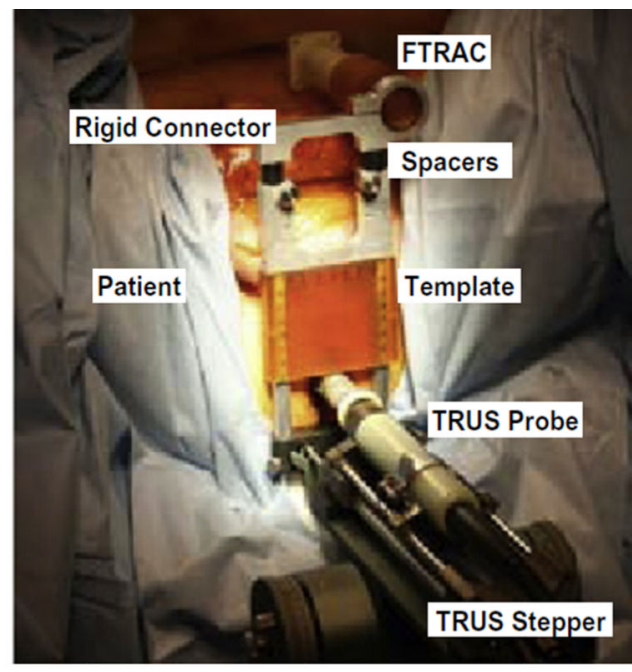

(c)

Fig. 10.

The FTRAC fiducial and the needle insertion template can be pre-calibrated using a rigid mount. (a) A CAD model of the FTRAC fiducial mounted on the seed-insertion needle template using a rigid connector. (b) An actual photograph of the FTRAC mounted on the template. (c) A zoomed annotated photograph of the clinical setup. 

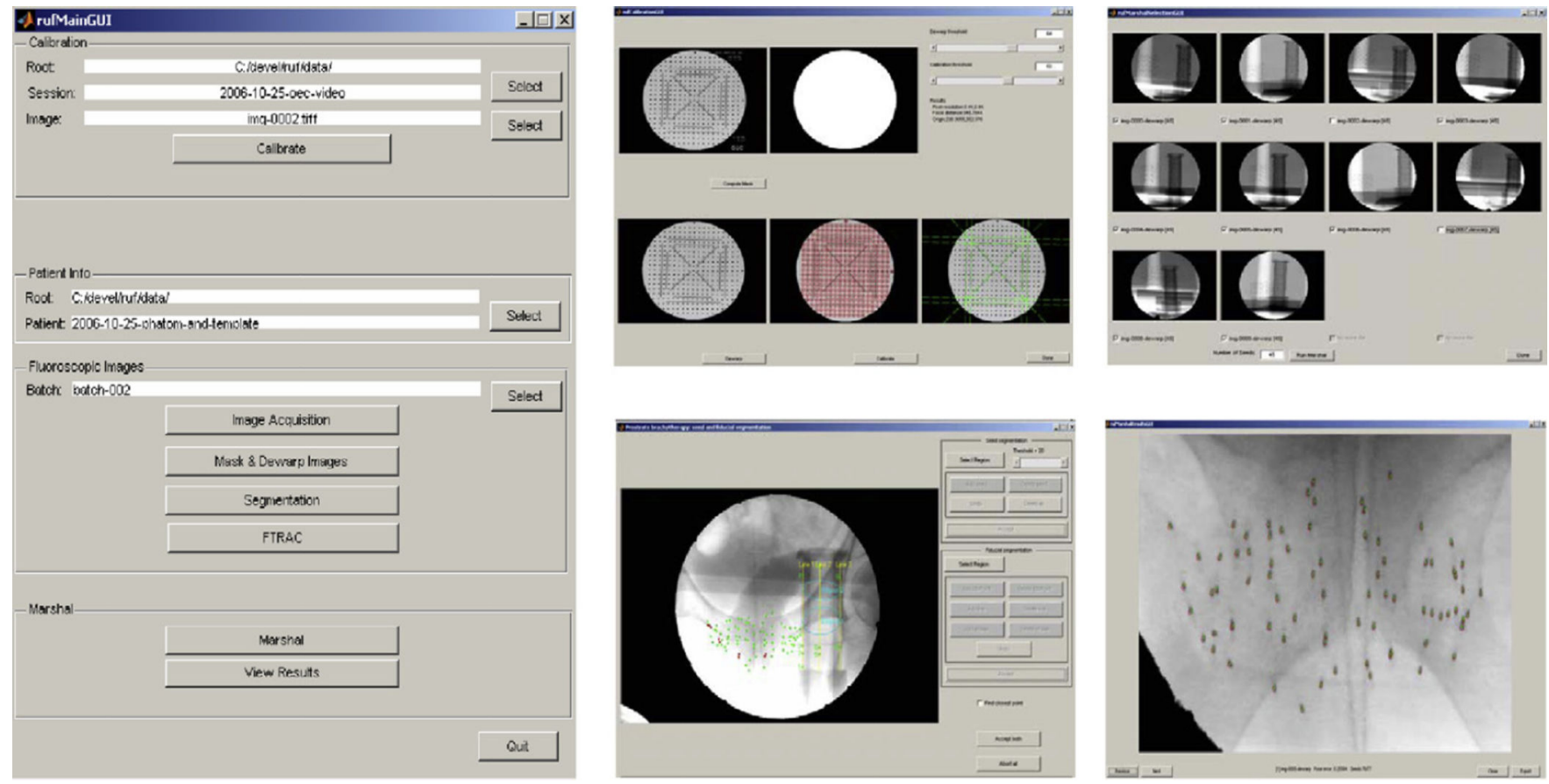

Fig. 11.

GUI screen captures of the main program, offline calibration, seed and fiducial segmentation, seed matching and validation of the reconstruction by back-projection. 


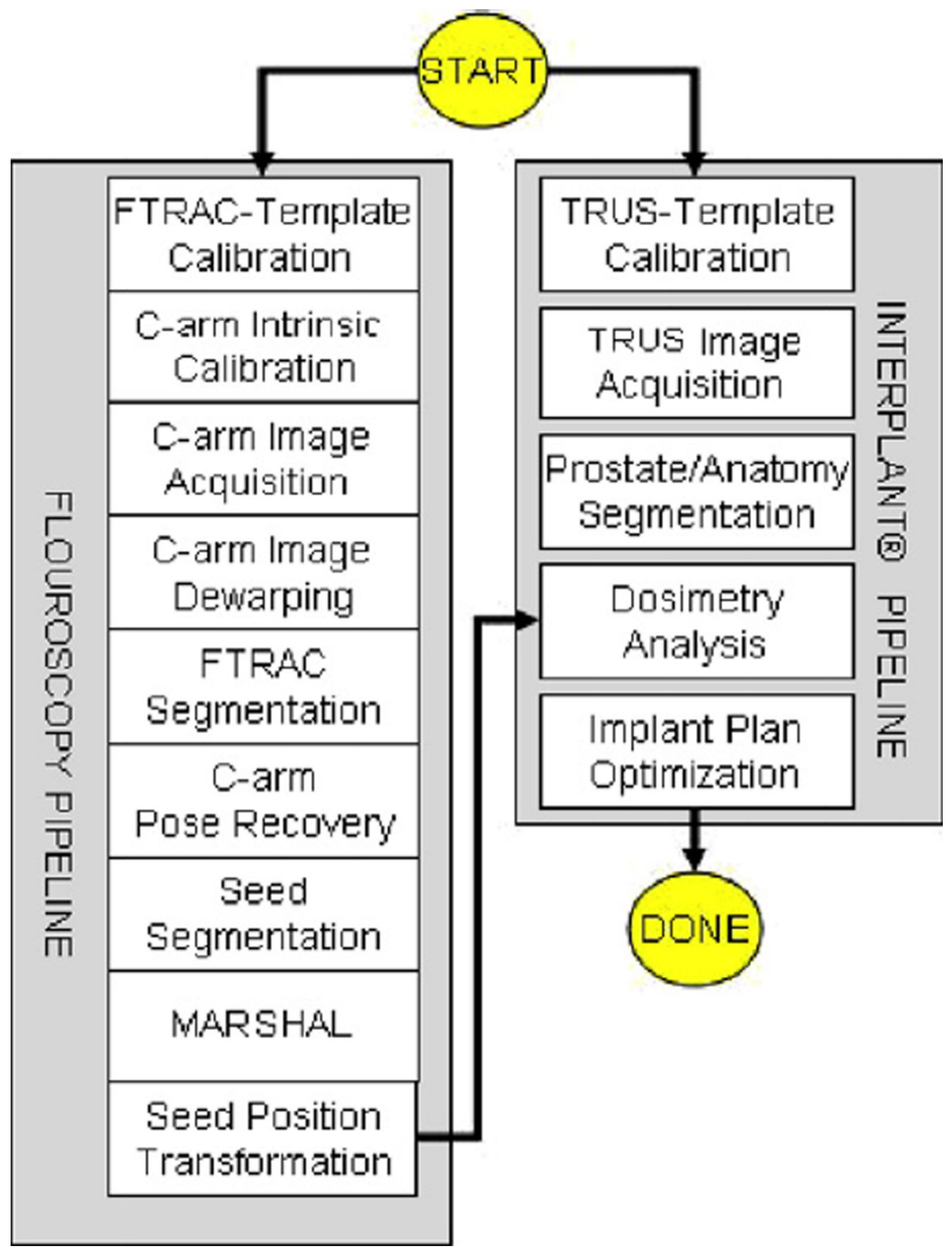

Fig. 12.

Workflow for intra-operative dosimetry and implant optimization. 


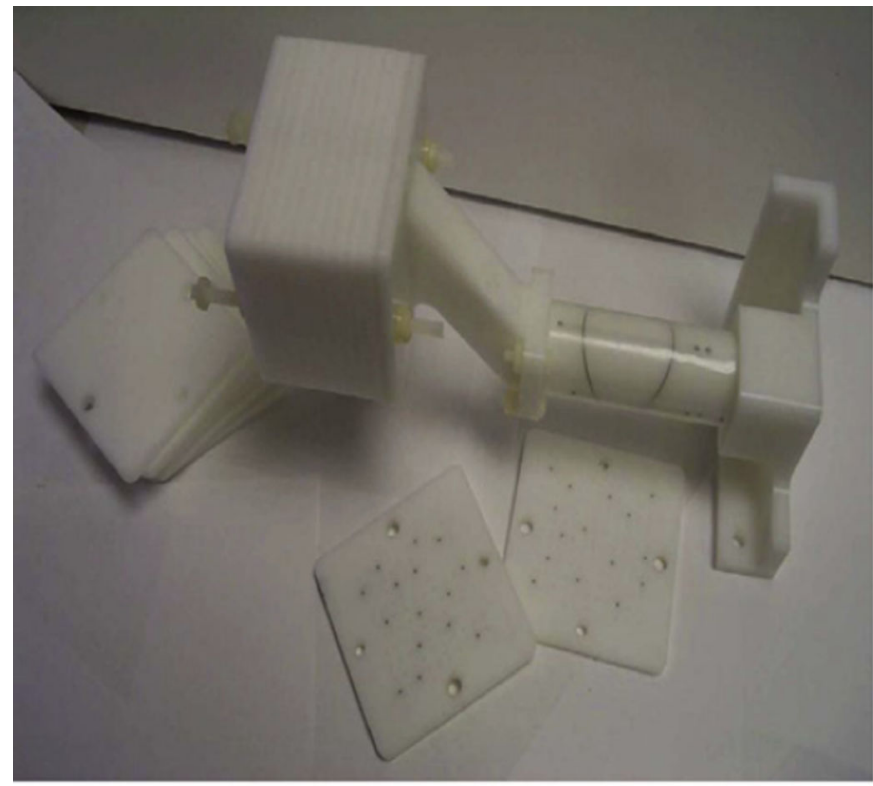

(a)

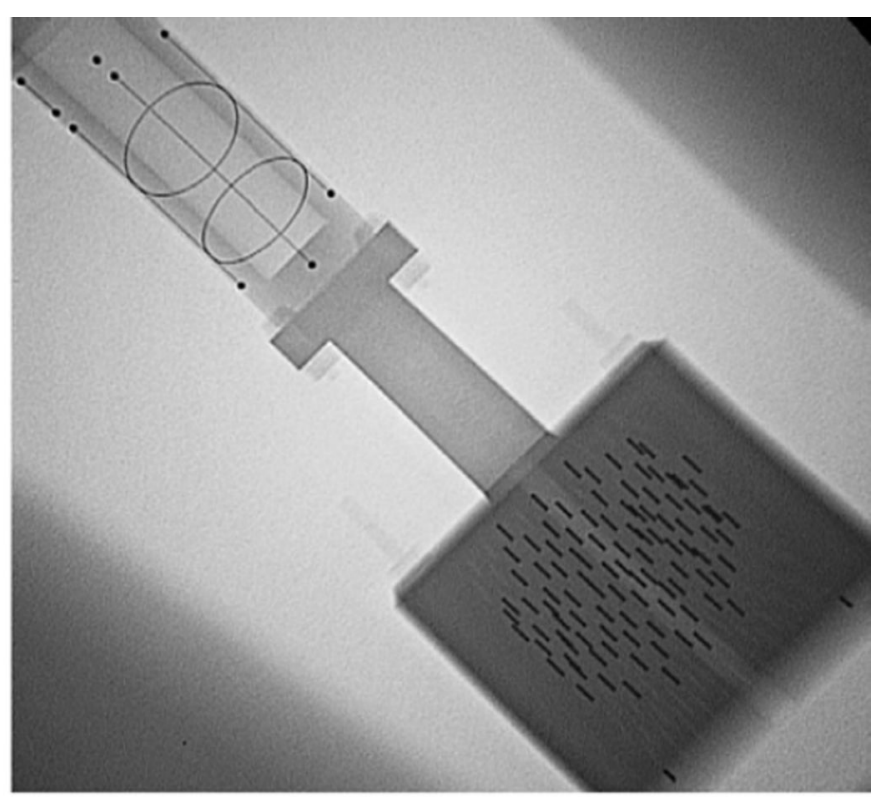

(b)

Fig. 13.

(a) An image of the seed phantom attached to the FTRAC fiducial. The phantom can replicate any implant configuration, using the twelve $5 \mathrm{~mm}$ slabs each with over a hundred holes. (b) A typical X-ray image of the combination. 


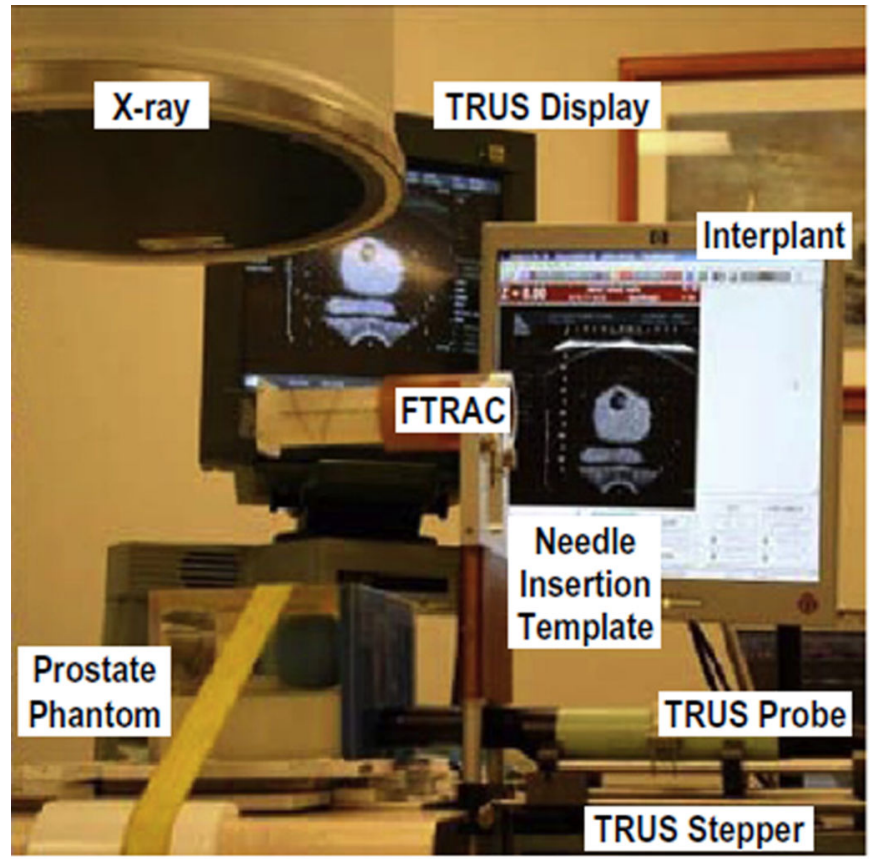

(a)

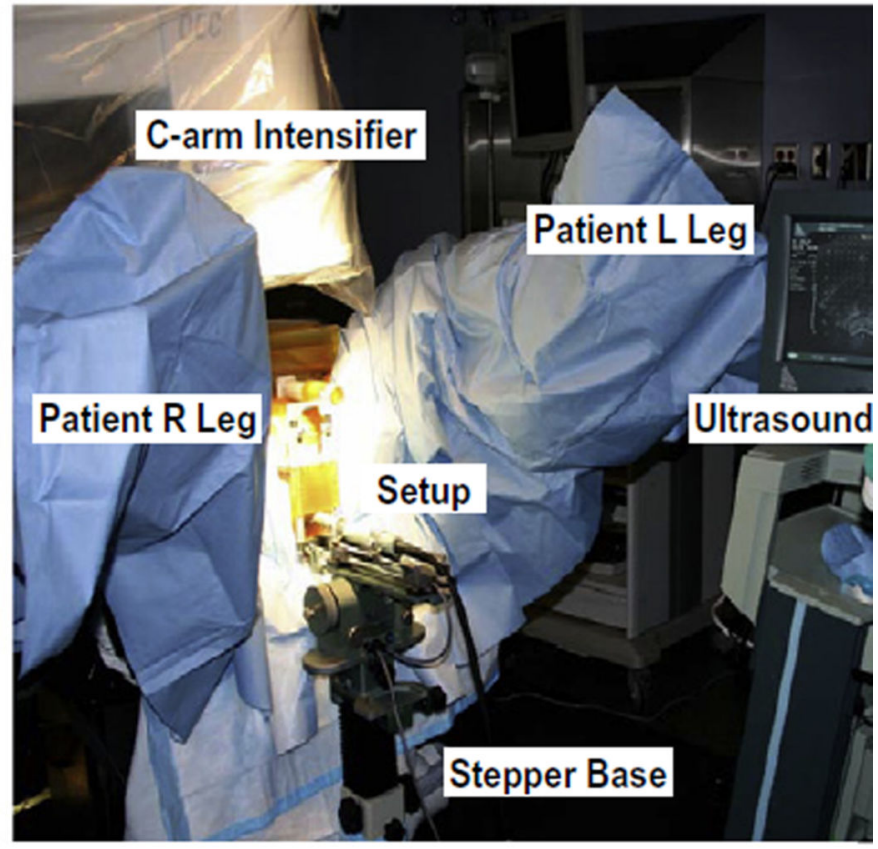

(b)

Fig. 14.

An annotated image of the (a) experimental setup for the training phantom experiments; (b) overall set up of the full operating area during the Phase-I clincal trials. 


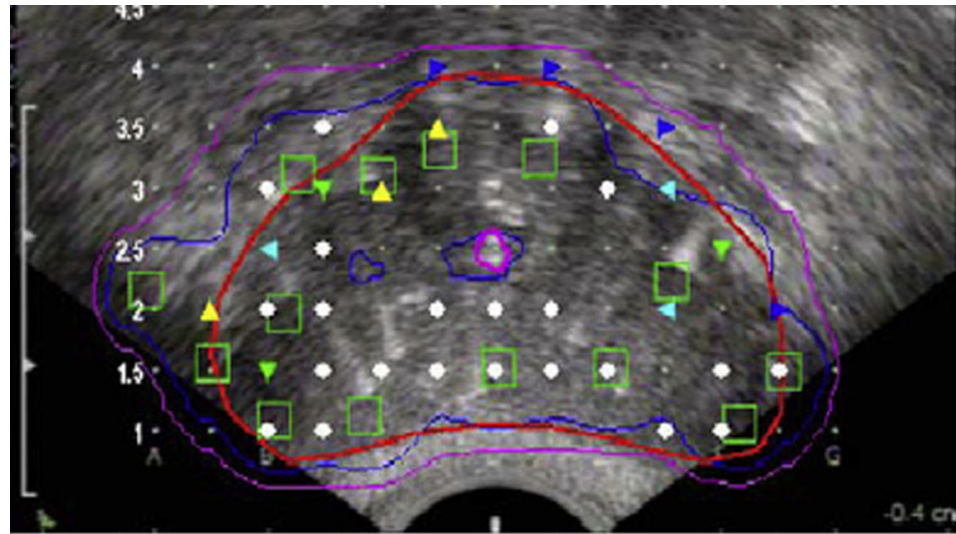

\section{Locations Without Guidance}

- 2 cold spots in this slice (yellow circles)

- 4 seeds drift out of slice (violet arrows)

- 2 seeds migrate in slice (orange arrows)

- Current seed locations (green boxes)

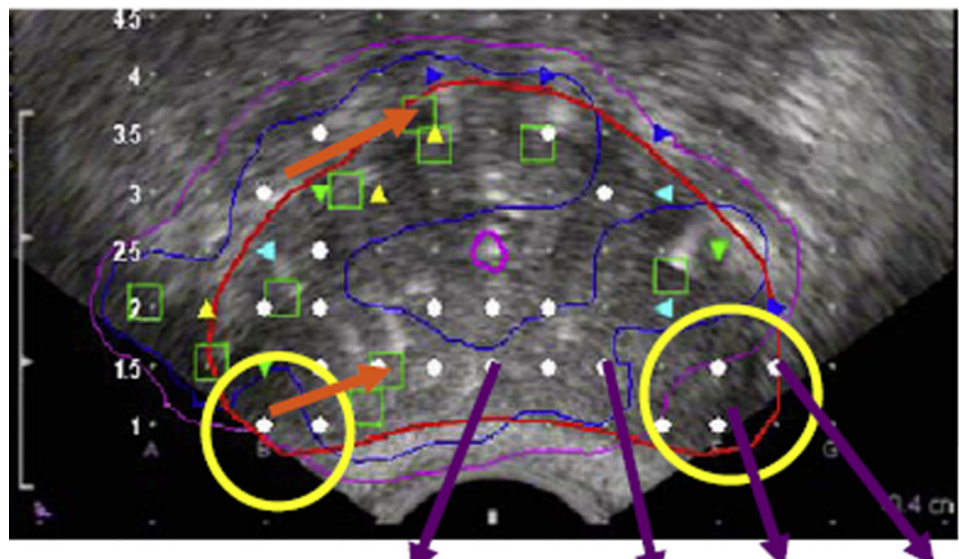

- Prostate contour

- $100 \%$ iso-dose contour (pink boundary)

- $150 \%$ iso-dose contour (blue boundary)

- Needles (white circ)

Locations With Guidance

Fig. 15.

The system is able to detect cold spots. The seed locations (and corresponding 100\%/150\% isodose contours) as assumed by the planning system (top) and as computed by the proposed system (bottom), discovering two cold spots in this slice. Four seeds have drifted out of the slice, while two have migrated significantly within. 

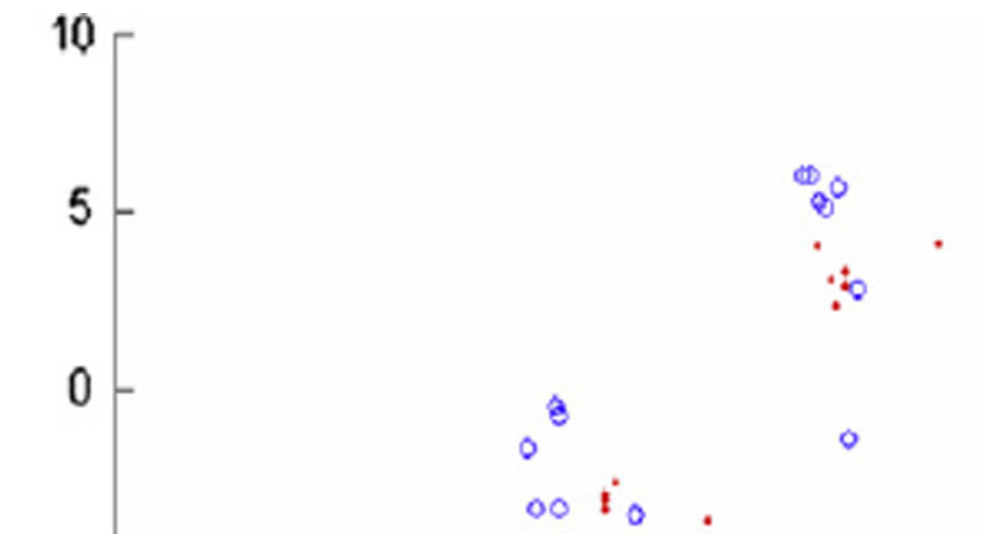

$\circ$

- 8
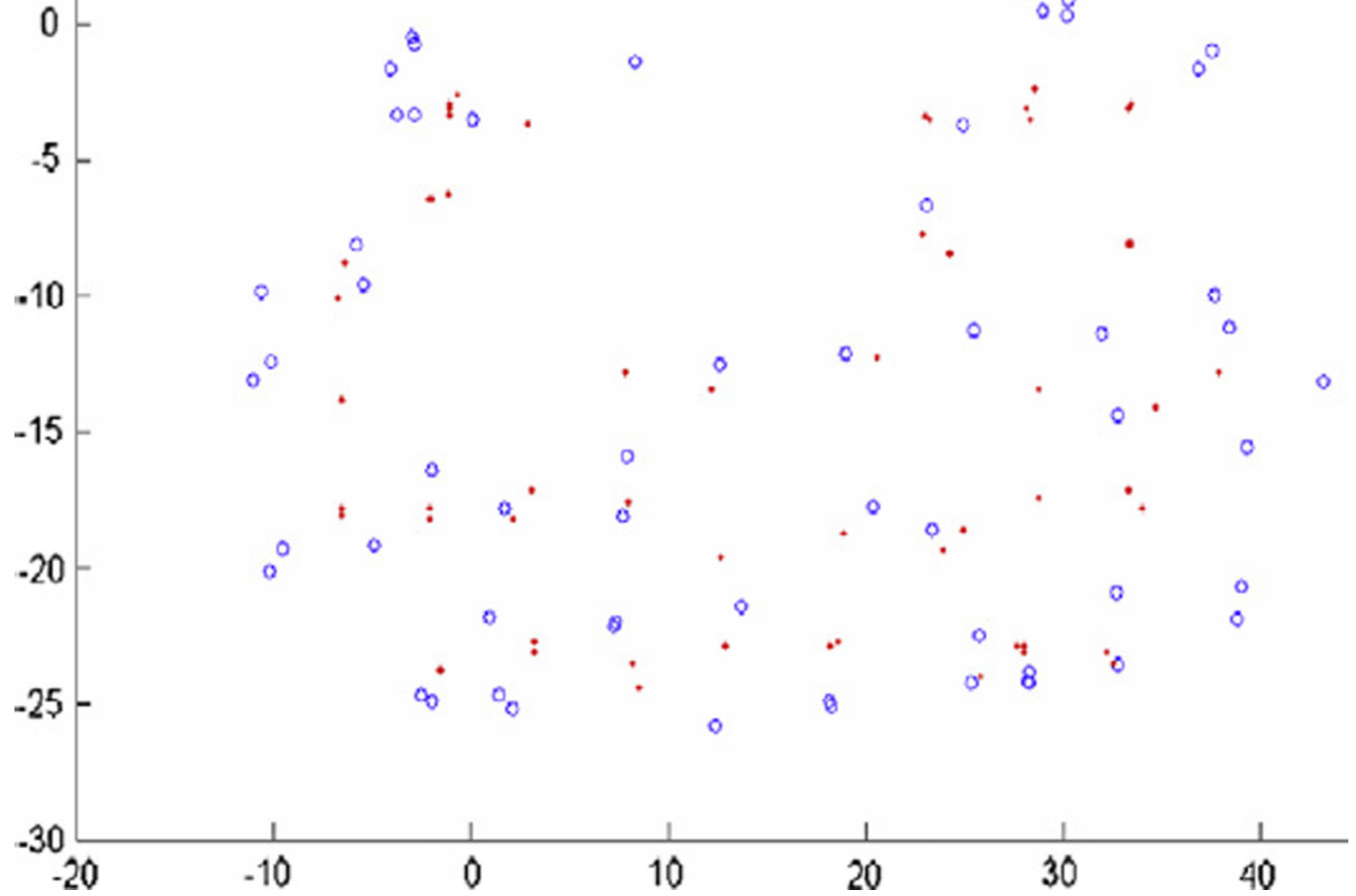

0

$\theta$

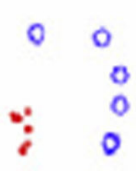

Blue Circle $=$ Actual X-ray

Fig. 16.

The system can visualize intra-operative edema, as seen for patient 3 (mean $4.6 \mathrm{~mm}$, STD $2.4 \mathrm{~mm}$, max $12.3 \mathrm{~mm}$ ). The 'planned' (red) vs. the 'reconstructed' (blue) seed positions as seen in the template view. A trend of outward dispersion from their initial locations is observed. 


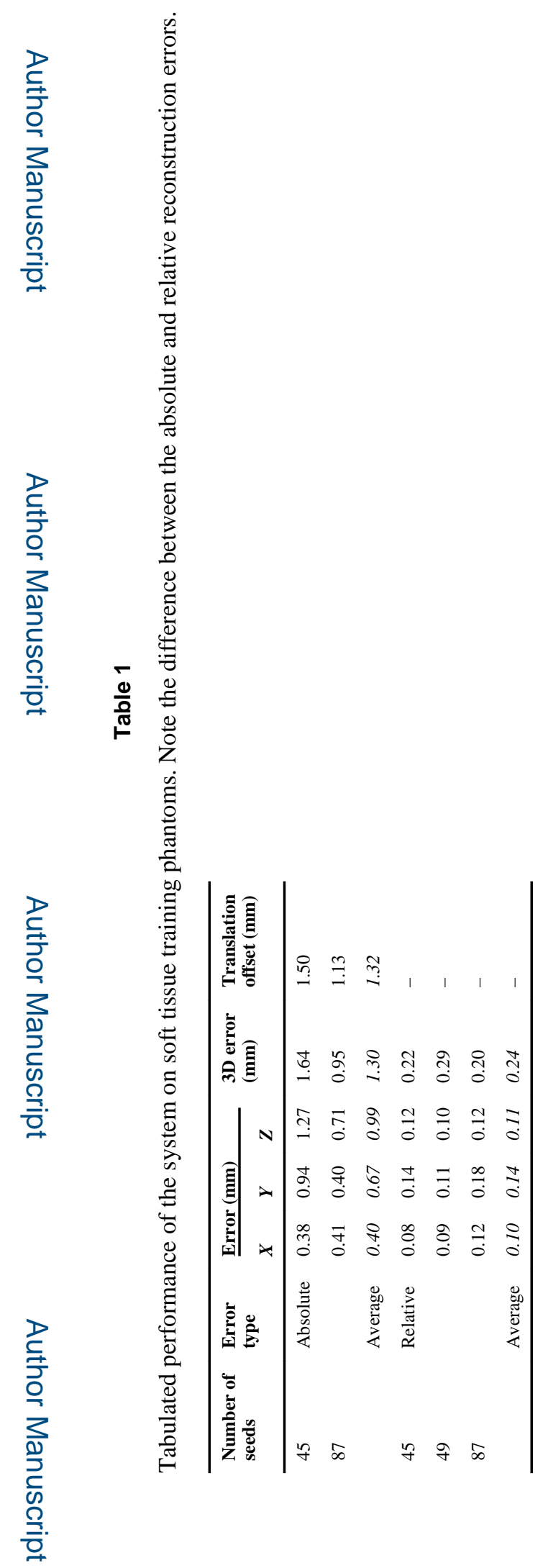

Med Image Anal. Author manuscript; available in PMC 2015 December 30. 


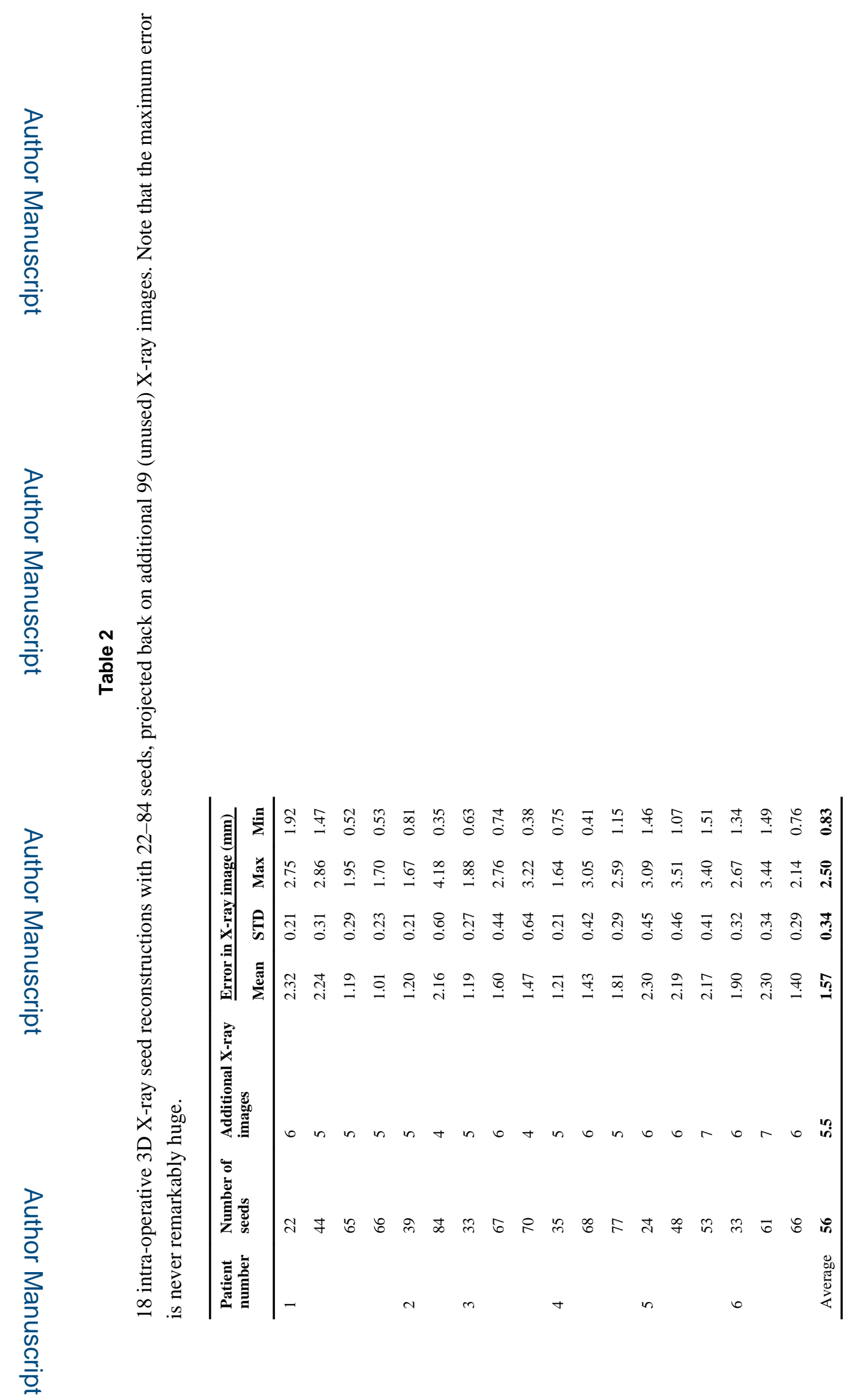

Med Image Anal. Author manuscript; available in PMC 2015 December 30. 


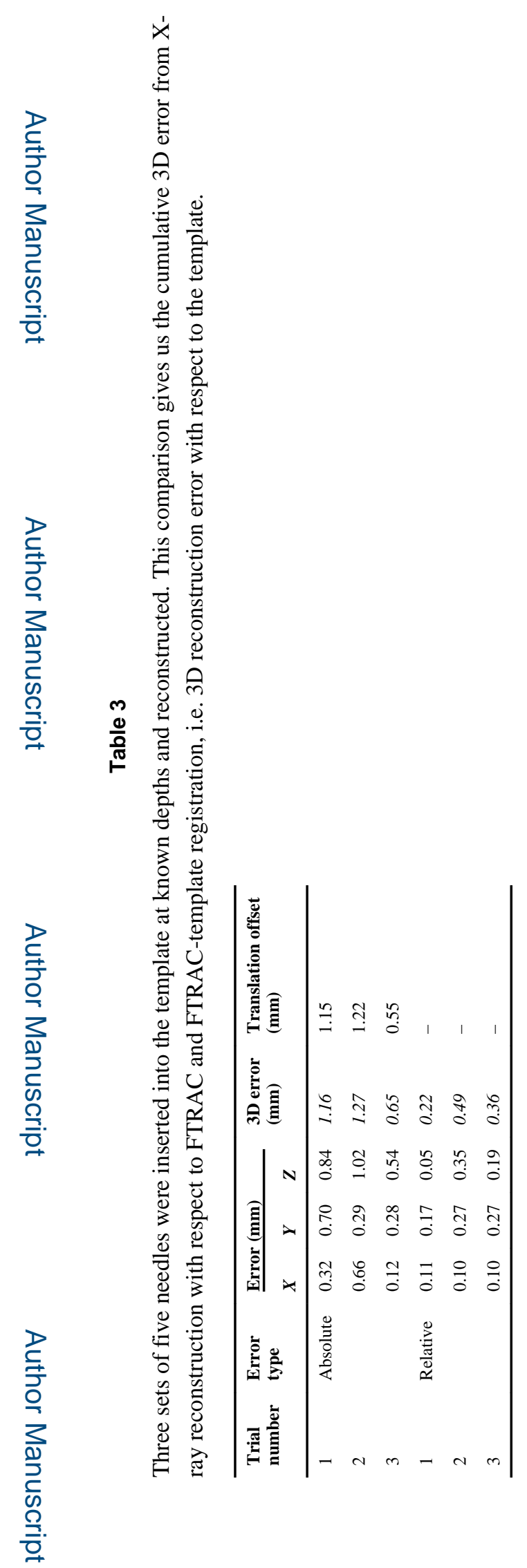

Med Image Anal. Author manuscript; available in PMC 2015 December 30. 


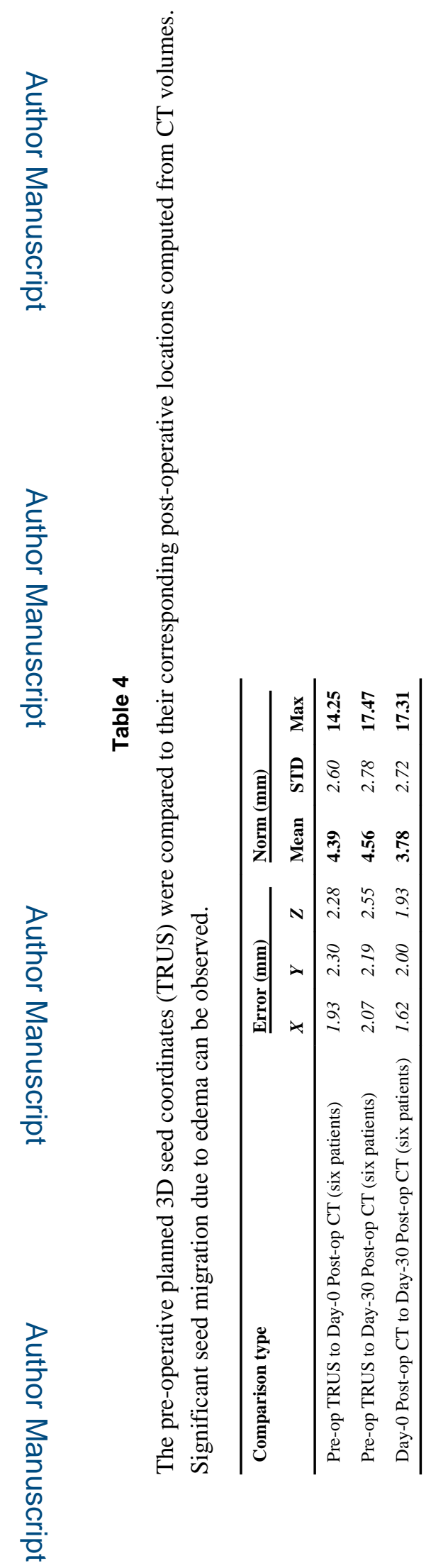

Med Image Anal. Author manuscript; available in PMC 2015 December 30. 


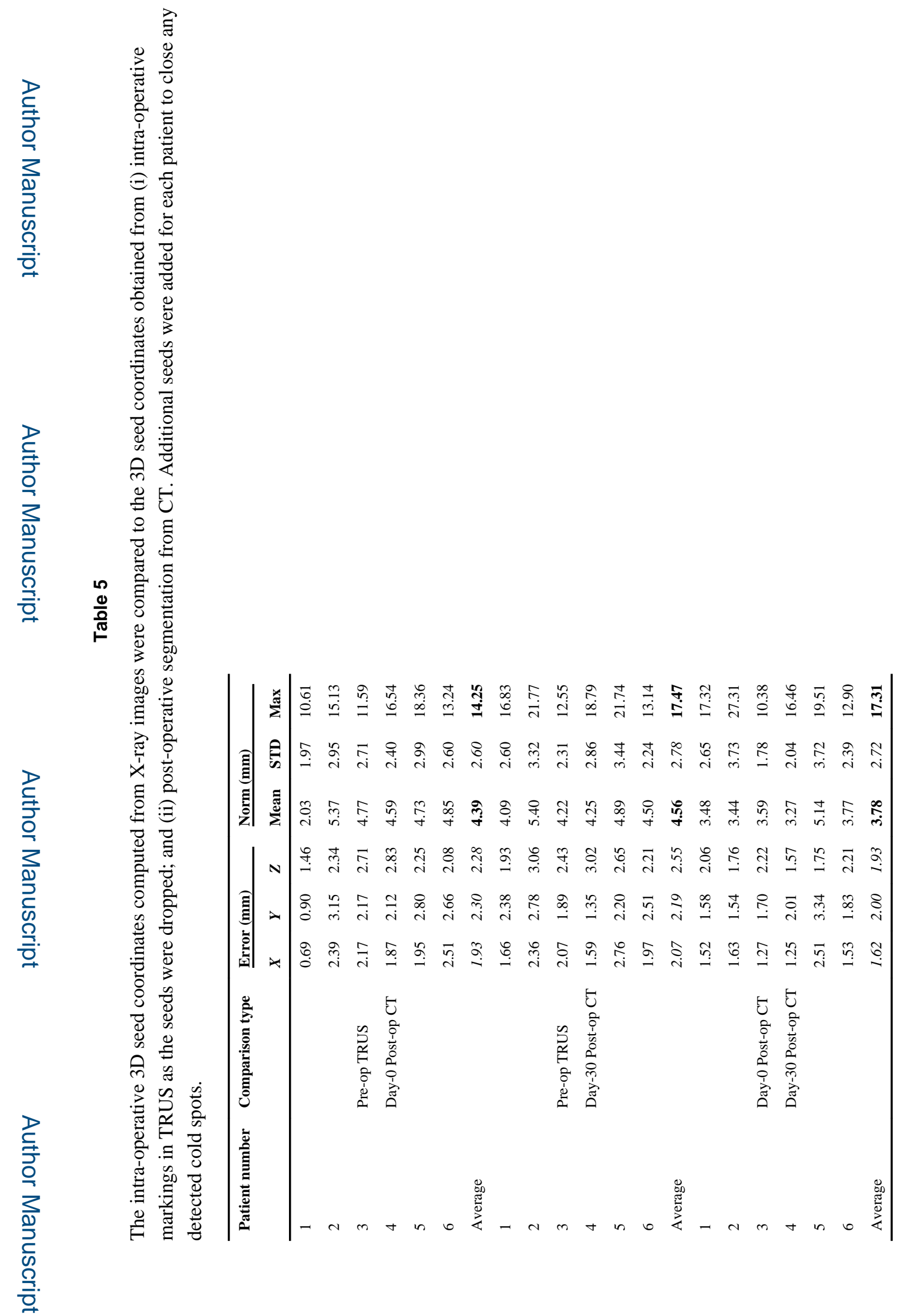

Med Image Anal. Author manuscript; available in PMC 2015 December 30. 


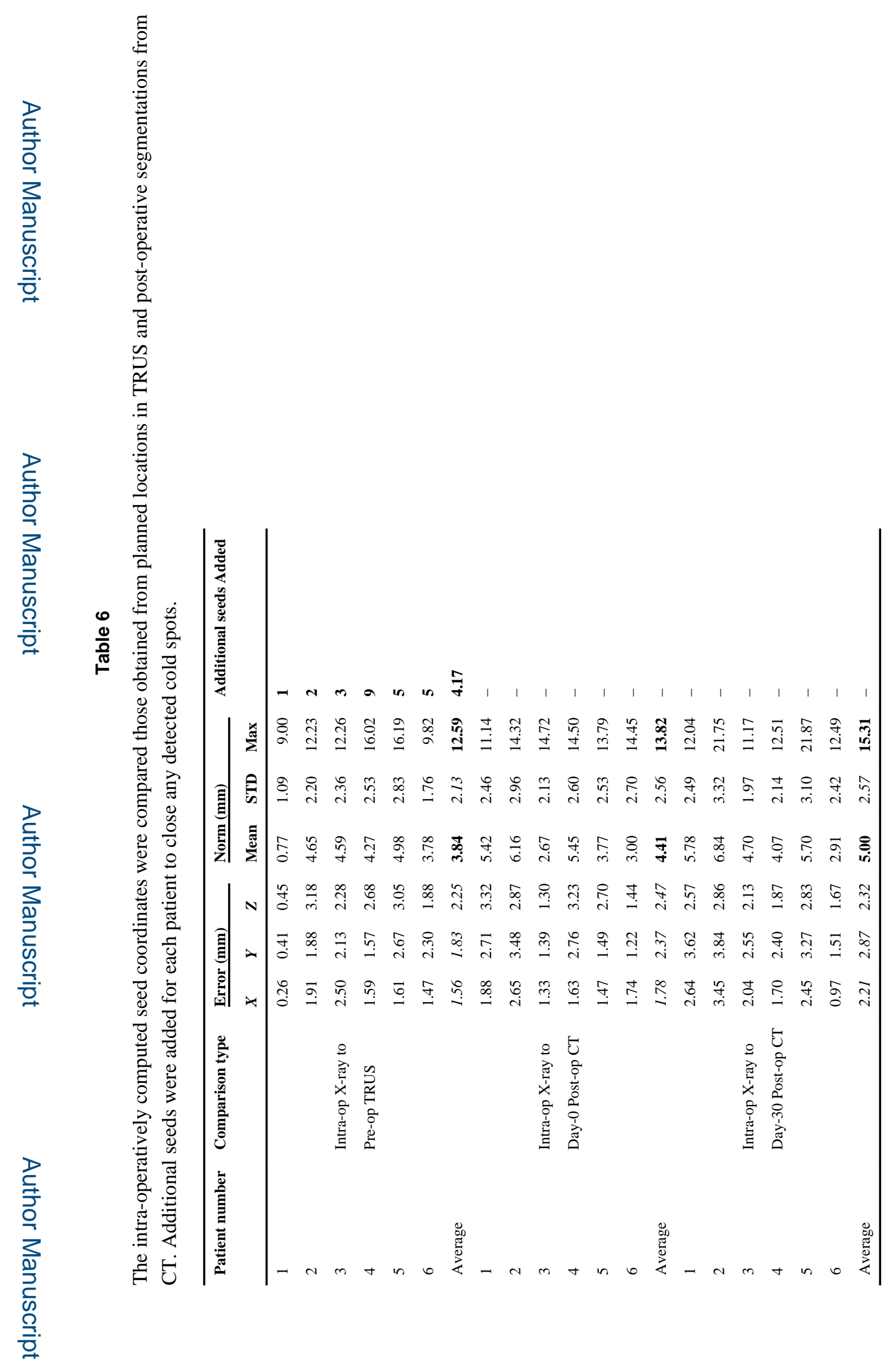

Med Image Anal. Author manuscript; available in PMC 2015 December 30. 